\title{
Constant Geodesic Curvature Curves and Isoperimetric Domains in Rotationally Symmetric Surfaces
}

\author{
MANUEL RitorÉ ${ }^{1}$
}

\begin{abstract}
In this paper we study curves with constant geodesic curvature in rotationally symmetric complete surfaces. Under monotonicity conditions on the Gauss curvature we classify the closed embedded ones in planes, cylinders, spheres and projective planes. We also distinguish the stable ones, i.e., the second order minima of perimeter while keeping constant the area enclosed. We prove existence and nonexistence of isoperimetric domains, and we show the isoperimetric domains when they exist.
\end{abstract}

\section{Introduction.}

In a Riemannian surface $M$ of area $A(M)$, the isoperimetric profile of $M$ is the function which assigns, to each positive value $A \leqslant A(M)$, the infimum of the perimeter of sets enclosing area $A$. If this value is attained by some set $\Omega$, then $\Omega$ is called an isoperimetric domain. To compute the isoperimetric profile of a given surface and to classify the isoperimetric domains when they exist are interesting and difficult global problems in Riemannian Geometry.

The classical approach to these problems is by means of isoperimetric inequalities, which are nothing but relations between the area of a set and its perimeter. The classical isoperimetric inequalities for surfaces of constant curvature allow us to find the isoperimetric domains in such surfaces, which are geodesic discs ([22], [18], [14]). Osserman's survey ([18]) covers the subject since the beginning with an exhaustive list of references. The one by Howards, Hutchings and Morgan ([14]) describes recent progress.

Until recently the isoperimetric profile was only known for the complete simply connected surfaces with constant Gauss curvature. In 1996 Benjamini and Cao ([3]) solved the isoperimetric problem in some rotationally symmetric planes by using the geodesic curvature flow on surfaces previously

\footnotetext{
${ }^{1}$ Work partially supported by DGICYT research group PB97-0785.
} 
studied by Grayson ([11]). From their results one can obtain a new isoperimetric inequality that has been explicitly stated and proved by P. Pansu for discs ([20]) and by Topping ([23]) and Howards, Hutchings and Morgan ([15]) for domains of general topological type. For planes of revolution with decreasing curvature and total positive curvature less than or equal to $2 \pi$ the isoperimetric domains are geodesic discs centered at the point of maximum curvature ([3]). For planes of revolution with decreasing curvature, projective planes with decreasing curvature, annuli with decreasing curvature and an end of finite area and some spheres the isoperimetric domains have been classified by Howards, Hutchings and Morgan ([15]).

In this paper we study curves with constant geodesic curvature in some rotationally symmetric surfaces by using methods of the Calculus of Variations. Our approach is different from the ones described above. Similar techniques to ours were employed by E. Schmidt ([22], [18, p. 1200]). Since an isoperimetric domain on a Riemannian surface has regular boundary, which is a closed smooth embedded curve with constant geodesic curvature ([2]), we can apply our results to find such optimal domains. Standard results in Geometric Measure Theory plus geometric arguments are used to prove existence and nonexistence of isoperimetric domains. We classify the closed embedded curves with constant geodesic curvature, we determine the stable ones, and we solve the isoperimetric problem in the following surfaces

(i) planes of revolution with decreasing curvature or increasing curvature;

(ii) spheres of revolution with an equatorial symmetry whose Gauss curvature is an increasing or decreasing function of the distance from the equator,

(iii) projective planes with decreasing or increasing curvature as a function of the distance from a given point, and

(iv) annuli with decreasing curvature such that the end with the largest curvature has finite area.

In planes with decreasing curvature, spheres with curvature increasing from the equator and in annuli with decreasing curvature the isoperimetric domains are bounded by circles of revolution. In a plane of revolution with strictly increasing curvature there are no stable closed embedded curves with constant geodesic curvature. Hence isoperimetric domains do not exist on such surfaces. The case of a sphere with curvature increasing from the equator is specially interesting since one has to construct first the boundaries 
of isoperimetric domains (they are not bounded by circles of revolution). It turns out that, in this case, isoperimetric domains are discs in a smooth family which collapses to a point in the equator when the area goes to zero. The isoperimetric domains in projective planes are obtained from the classification of constant geodesic curvature curves in the covering spheres. Annuli with monotone curvature (without the finite area assumption for the end with the largest curvature) cannot be studied with the methods of this paper.

The initial motivation for this work was a paper by S. Montiel ([17]) treating hypersurfaces with constant mean curvature in manifolds $M^{n}, n \geqslant$ 3 , foliated by totally umbilical hypersurfaces. The results in [17], extensions of the classical Theorems by Liebmann and Alexandrov for constant mean curvature surfaces in $\mathbb{R}^{3}$, do not extend to curves in surfaces. In fact there are strong differences between the cases $n=2$ and $n \geqslant 3$. Liebmann's Theorem, as proved in [17], is local in nature (only the geometry of the ambient manifold about the hypersurface is involved) and it is valid for manifolds with singularities. For surfaces, such theorem is of a global nature, and it is no longer true if the surface has a singularity.

We have organized this paper in several sections. In the preliminaries one we state general results that will be used along the paper. In the following sections we consider the different types of surfaces: planes in the second one, spheres in the third section, projective planes in the fourth one and annuli in the last one.

By means of the techniques used in this paper also tori, Klein bottles and symmetric annuli (of catenoid type) can be studied. Since additional difficulties arise in these cases we shall treat them in a forthcoming paper $([21])$.

Finally we made some advice about terminology. A function $f: \mathbb{R} \rightarrow \mathbb{R}$ will be called decreasing (resp. increasing) if $f(x) \geqslant f(y)$ (resp. $f(x) \leqslant$ $f(y)$ ) whenever $x<y$. If the inequality is strict the function will be called strictly decreasing (resp. strictly increasing). A monotone function is one which is either increasing or decreasing. A rotationally symmetric surface will mean a surface endowed with a one-parameter group of isometries. The poles of a rotationally symmetric surface are the points which are fixed by all the isometries of the compact one-parameter group. Computing the index of the Killing field at the poles it can be easily checked that all these surfaces have nonnegative Euler characteristic. The reader is referred to [17] for more results and references on rotationally symmetric surfaces.

The author wishes to thank Frank Morgan for many interesting discussions and for suggesting some improvements in the paper. 


\section{Preliminaries.}

Consider the product $\mathbb{S}^{1} \times I$, where $\mathbb{S}^{1}$ is the unit circle and $I \subset \mathbb{R}$ is an interval, endowed with the Riemannian metric

$$
d s^{2}=d t^{2}+f(t)^{2} d \theta^{2}
$$

for $\theta \in \mathbb{S}^{1}$ and $t \in I$. This Riemannian surface is a warped product.

Over this surface we define the vector field $X=f(t) \partial_{t}$, which is conformal ([17]). Let $D$ be the Riemannian connection associated to the metric $d s^{2}$. From (1.1) it can be easily proved that $D_{u} X=f^{\prime}(t) u$ for any tangent vector $u$ to $M$, where primes denote derivatives with respect to $t$. It follows

$$
\operatorname{div} X=2 f^{\prime}(t),
$$

where div is the divergence of the vector field $X$.

The Gauss curvature of the metric depends only on $t$ and it is given by

$$
K(t)=-\frac{f^{\prime \prime}(t)}{f(t)} .
$$

The geodesic curvature of the circle $\mathbb{S}^{1} \times\{t\}$, computed with respect to the normal $-\partial_{t}$, is given by

$$
h(t)=\frac{f^{\prime}(t)}{f(t)},
$$

The length of the closed curve $\mathbb{S}^{1} \times\{t\}$ is given by

$$
L(t)=2 \pi f(t) .
$$

A fundamental observation, to be used later, is that the function of $t$

$$
\left(f^{\prime}\right)^{2}-f f^{\prime \prime}=(2 \pi)^{-2} L^{2}\left(K+h^{2}\right),
$$

has, up to a positive function, the same derivative with respect to $t$ that the Gauss curvature $K(t)$. Hence $K(t)$ and $L^{2}\left(K+h^{2}\right)(t)$ are simultaneously increasing or decreasing and they have the same critical points.

Consider a curve $\gamma(s)=(\theta(s), t(s))$ parameterized by arc-length $s$. The tangent vector to $\gamma$ will be denoted by $d \gamma / d s$. Assume that the surface is oriented by $d t \wedge d \theta$ and let $\sigma$ be the oriented angle $\angle\left(\partial_{t}, d \gamma / d s\right)$. Then $d \gamma / d s$ is given by

$$
\frac{d \gamma}{d s}=\left(\frac{d \theta}{d s}, \frac{d t}{d s}\right)=\frac{\sin \sigma}{f(t)} \partial_{\theta}+\cos \sigma \partial_{t} .
$$


We consider the unit normal vector field $N$ to $\gamma$ given by

$$
N=\frac{\cos \sigma}{f(t)} \partial_{\theta}-\sin \sigma \partial_{t}
$$

From (1.1) we easily see

$$
D_{d \gamma / d s} \frac{d \gamma}{d s}=\left\{\frac{d \sigma}{d s}+\frac{f^{\prime}(t)}{f(t)} \sin \sigma\right\} N
$$

So we conclude

Proposition 1.1. If $\gamma(s)=(\theta(s), t(s))$ is a curve parameterized by arclength $s$ with geodesic curvature $h$, computed with respect to the normal $\cos \sigma / f(t) \partial_{\theta}-\sin \sigma \partial_{t}$, then $\theta(s), t(s), \sigma(s)$ satisfy the following system of ordinary differential equations

$$
\begin{aligned}
& \frac{d t}{d s}=\cos \sigma \\
& \frac{d \theta}{d s}=\frac{\sin \sigma}{f(t)}, \\
& \frac{d \sigma}{d s}=h-\frac{f^{\prime}(t)}{f(t)} \sin \sigma, \text { nonumber }
\end{aligned}
$$

Moreover, if $h$ is constant then, for $c$ in the closure of the domain of definition of $f$, the function

$$
f(t) \sin \sigma-h \int_{c}^{t} f(\xi) d \xi
$$

is constant over any solution of $(*)$.

Proof. It only remains to check that $(* *)$ is constant over solutions of $(*)$, which is immediate differentiating with respect to $s$.

The function $(* *)$ is usually called a first integral of $(*)$ in the terminology of the Calculus of Variations. First integrals are usually obtained from Noether's Theorem ([9]). For $h=0$ the expression $f(t) \sin \sigma=$ constant is nothing but Clairaut relation for geodesics in surfaces of revolution.

We shall usually speak of a solution $\gamma=(\theta, t)$ to $(*)$ since the angle $\sigma$ is determined by $\theta$ and $t$. We define the energy $E$ of a parameterized curve $\gamma$, 
solution to $(*)$ with $h$ constant, to be the constant value given by $(* *)$ over $\gamma$.

From the uniqueness of solutions to $(*)$ with respect to the initial conditions we easily obtain

Proposition 1.2. Let $\gamma=(\theta, t)$ be a solution to $(*)$ with constant geodesic curvature $h$. Then

(i) If $(d t / d s)\left(s_{0}\right)=0$ then $\gamma$ is symmetric with respect to the geodesic $\theta=\theta\left(s_{0}\right)$. More precisely $t\left(s_{0}-s\right)=t\left(s_{0}+s\right), \theta\left(s_{0}-s\right)=2 \theta_{0}-\theta\left(s_{0}+s\right)$, and $\sigma\left(s_{0}-s\right)=\pi-\sigma\left(s_{0}+s\right)$.

(i) The curve $\gamma$ can be translated along the $\theta$-axis. More precisely, $\theta(s)+a$, $t(s), \sigma(s)$ is a solution to $(*)$ for any real a.

The behavior of solutions to $(*)$ with $h$ constant is described in next Proposition

Proposition 1.3. Let $\gamma=(\theta, t)$ be a solution to $(*)$ with $h$ constant such that $t\left(s_{0}\right)$ is a strict maximum of the $t$-coordinate and $\sin \sigma\left(s_{0}\right)=1$. Assume that $t\left(s_{1}\right)$, for $s_{1}>s_{0}$, is a critical point of the $t$-coordinate and that there are no more critical points of $t$ in the interval $\left(s_{0}, s_{1}\right)$. Then we have

(i) If $\sin \sigma\left(s_{1}\right)=1$ then $\gamma$ is a graph over $\theta$ which is periodic in $\theta$. The curve $\gamma$ yields a closed embedded curve if and only if the $\theta$-distance between two consecutive maxima or minima of the $t$-coordinate equals $2 \pi / k$, for some $k \in \mathbb{N}$.

(ii) If $\sin \sigma\left(s_{1}\right)=-1$ then there is a point of vertical tangent vector for some $s \in\left(s_{0}, s_{1}\right)$. The curve $\gamma$ yields a closed embedded curve only if $\theta\left(s_{0}\right)=\theta\left(s_{1}\right)$.

Proof. The function $t$ is strictly decreasing for $s>s_{0}$ close enough to $s_{0}$. As there are no critical points of $t$ in $\left(s_{0}, s_{1}\right)$ we conclude that $t$ is decreasing in this interval. Choosing $c<t\left(s_{1}\right)$ in $(* *)$ we observe that $f(t) \sin \sigma$ is strictly monotone or constant as a function of $t$.

In case (i) $\sin \sigma\left(s_{1}\right)=1$ and so $f(t) \sin \sigma>0$ for all $s \in\left(s_{0}, s_{1}\right)$. This implies that $d \theta / d s>0$ and so the curve is a periodic graph over $\theta$. It is then closed and embedded trivially if its minimum $\theta$-period is exactly $2 \pi / k$, for $k \in \mathbb{N}$.

In case (ii) $\sin \sigma\left(s_{1}\right)=-1$ and, since $f(t) \sin \sigma$ is strictly monotone as a function of $t$, there is exactly one $s \in\left(s_{0}, s_{1}\right)$ with tangent vector $-\partial_{t}$. 
Reflecting with respect to the extrema of $t$ it follows easily that $\gamma$ yields an embedded curve only if $\theta\left(s_{0}\right)=\theta\left(s_{=} 1\right)$.

The curves described in the above Proposition have the same behavior as the ones obtained by Delaunay $([6])$ as the generating curves of surfaces of revolution with nonzero constant mean curvature in $\mathbb{R}^{3}$. By this analogy the curves with constant geodesic curvature which are graphs over $\theta$ will be referred to as unduloid type curves or unduloids, and the ones whose tangent vector is vertical somewhere as nodoid type curves or nodoids.

These curves are depicted in Figure 1.

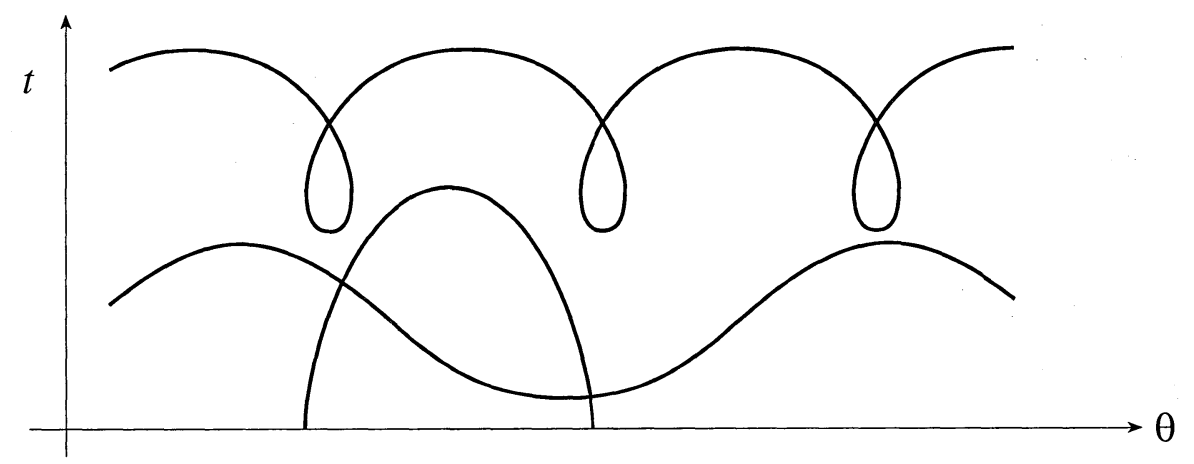

Figure 1: Nodoid and unduloid type solutions to equations $(*)$.

The following two lemmas are key results to find the curves with constant geodesic curvature. The proof of the first one is just a direct computation using equations $(*)$. The second one follows from the proof of Sturm's Separation Theorem ([13, Corollary 3.1]) and Osserman ([19])

Lemma 1.4. Let $\gamma=(\theta, t)$ be a solution to $(*)$ with constant geodesic curvature. Let $u=\cos \sigma$. When $d \theta / d s \neq 0$ we have

$$
\frac{d^{2} u}{d \theta^{2}}+\left[\left(f^{\prime}\right)^{2}-f f^{\prime \prime}\right] u=0 .
$$

Lemma 1.5. Let $u_{1}, u_{2}:[a, b] \rightarrow \mathbb{R}$ be solutions to

$$
u_{1}^{\prime \prime}+g_{1} u_{1}=0, \quad u_{2}^{\prime \prime}+g_{2} u_{2}=0,
$$

where primes denote the derivative with respect to the parameter in $[a, b]$. Assume that $u_{1}, u_{2}>0$ at some small interval $(a, a+\varepsilon)$ on $(a, b)$, that 
$\left(u_{1}^{\prime} u_{2}-u_{1} u_{2}^{\prime}\right)(a) \geqslant 0$, and that $g_{2} \geqslant g_{1}$ in $[a, b]$. Then the first zero $b^{\prime}$ of $u_{2}$ is less than or equal to the first zero of $u_{1}$. If they coincide then $\left(u_{1}^{\prime} u_{2}-u_{1} u_{2}^{\prime}\right)(a)=0$ and $g_{1} \equiv g_{2}$ on $\left[a, b^{\prime}\right]$.

If we assume $u_{1}, u_{2}>0$ on $(a, b),\left(u_{1}^{\prime} u_{2}-u_{1} u_{2}^{\prime}\right)(a) \geqslant 0, g_{2} \geqslant g_{1}$, and the limit $M=\lim _{t \rightarrow 0} u_{1}(t) / u_{2}(t)$ is finite then $u_{1} \geqslant M u_{2}$ on $(a, b)$. Moreover, if $u_{2}(b)=M u_{1}(b)$ then $\left(u_{1}^{\prime} u_{2}-u_{1} u_{2}^{\prime}\right)(a)=0$ and $g_{1} \equiv g_{2}$ on $[a, b]$.

Curves with constant geodesic curvature in a Riemannian surface $M$ are the critical points of length under the restriction that the area enclosed by the curve is constant. If the boundary of a relatively compact domain has several connected components and it is a critical point of length under the above area constraint then all the boundary components have the same constant geodesic curvature measured with respect to the inner normal ([2], [18]). We shall say that a curve $C$ enclosing a set $\Omega$ is stable if it has constant geodesic curvature with respect to the inner normal and the second derivative of length for variations keeping constant the area enclosed is nonnegative. An unstable curve is a non stable one. A curve enclosing a domain is two-sided since one can choose a normal vector to the curve pointing to the domain. Analytically a two-sided curve $C$ is stable if and only if

$$
I(u)=-\int_{C} u\left\{\frac{d^{2} u}{d s^{2}}+\left(K+h^{2}\right) u\right\} d s \geqslant 0,
$$

for all functions $u: C \rightarrow \mathbb{R}$ such that $\int_{C} u d s=0([2])$. In the above formula $K$ is the Gauss curvature of $M, h$ is the geodesic curvature of $C, d / d s$ is the derivative with respect to arc-length on $C$ and $d s$ is the Riemannian measure on $C$. So $d^{2} / d s^{2}$ is the one-dimensional Laplacian. The left side of (1.4) is the quadratic form, often called the index form, associated to the self-adjoint operator

$$
J(u)=\frac{d^{2} u}{d s^{2}}+\left(K+h^{2}\right) u,
$$

which will be referred to as the Jacobi operator or the second variation operator. Associated to each connected component $C^{\prime}$ of the curve there is an increasing sequence of eigenvalues $\left\{\lambda_{i}\left(C^{\prime}\right)\right\}_{i \in \mathbb{N}}$. We refer to the reader to Chavel's book ([4]) for standard properties of eigenvalues. A Jacobi field $u$ is a solution to the equation $J(u)=0$. From $(*)$ one can prove that $u=f(t) \cos \sigma$ is a Jacobi field over any solution $\gamma$ to $(*)$. This function is the normal component of the Killing field $\partial_{\theta}$ restricted to $\gamma$.

If a connected curve is stable then at most the first eigenvalue is negative. If $C_{1}, C_{2}$ are connected curves with the same constant geodesic curvature $h$, 
$\lambda_{1}\left(C_{1}\right), \lambda_{1}\left(C_{2}\right) \leqslant 0$, and one of them is negative then $C_{1} \cup C_{2}$ is an unstable curve.

The notion of stability is related to the isoperimetric problem since the boundary of an isoperimetric domain is a stable curve. Of course each curve contained in a stable one is also a stable one.

The following result characterizes the stability of the closed curves $\mathbb{S}^{1} \times$ $\{t\}$ in the rotationally symmetric surfaces we introduced at the beginning of the section.

Lemma 1.6. For $t$ fixed, the curve $\mathbb{S}^{1} \times\{t\}$ is stable if and only if

$$
\left[\left(f^{\prime}\right)^{2}-f f^{\prime \prime}\right](t) \leqslant 1, \quad \text { or equivalently } \quad L^{2}\left(K+h^{2}\right)(t) \leqslant 4 \pi^{2} .
$$

Proof. The curve $\mathbb{S}^{1} \times\{t\}$ is isometric to the circle of radius $f(t)$ and the function $K+h^{2}$ equals $-f^{\prime \prime} / f+\left(f^{\prime} / f\right)^{2}$, which is a constant function over $\mathbb{S}^{1} \times\{t\}$. The stability of the curve is then equivalent ([1]) to that the first nonzero eigenvalue $\lambda_{2}=1 / f^{2}(t)$ of the Laplacian of the curve is greater than or equal to $\left[-f^{\prime \prime} / f+\left(f^{\prime} / f\right)^{2}\right](t)$, which implies (1.6).

We now write down the condition for the boundary of an annulus to be stable.

Lemma 1.7. The boundary of the annulus $\Omega=\mathbb{S}^{1} \times\left[t_{1}, t_{2}\right]$ is stable if and only if each connected component $\partial \Omega_{1}=\left\{t=t_{1}\right\}, \partial \Omega_{2}=\left\{t=t_{2}\right\}$ is separately stable and

$$
\frac{K+h^{2}}{L}\left(t_{1}\right)+\frac{K+h^{2}}{L}\left(t_{2}\right) \leqslant 0 .
$$

Moreover if $f(t)=f(-t)$ for all $t$ and $t_{1}=-t_{2}$ then the annulus $\mathbb{S}^{1} \times\left[-t_{2}, t_{2}\right]$ is stable if and only if

$$
\left(K+h^{2}\right)\left(t_{2}\right) \leqslant 0 .
$$

Proof. If $\partial \Omega$ is stable then $\partial \Omega_{i}$ is also stable for $i=1,2$. Take the function

$$
u= \begin{cases}-L\left(t_{2}\right), & \partial \Omega_{1}, \\ L\left(t_{1}\right), & \partial \Omega_{2},\end{cases}
$$


which has mean zero when integrated over $\partial \Omega$. Inserting this function in the index form we obtain $Q(u, u) \geqslant 0$ by stability. Inequality (1.7) then follows immediately.

Suppose now that each connected component $\partial \Omega_{i}, i=1,2$, is stable and that inequality (1.7) holds. Let $u: \partial \Omega \rightarrow \mathbb{R}$ be a mean zero function. Call $u_{i}, i=1,2$, to the restriction of $u$ to $\partial \Omega_{i}$. Then $u_{i}=c_{i}+v_{i}$, where $c_{i}$ is a constant and the integral of $v_{i}$ over $\partial \Omega_{i}$ is zero. We have

$$
Q\left(u_{i}, u_{i}\right)=Q\left(c_{i}, c_{i}\right)+Q\left(v_{i}, v_{i}\right)+2 Q\left(c_{i}, v_{i}\right) .
$$

Note that $Q\left(c_{i}, v_{i}\right)=0$ since $c_{i}$ is constant and $v_{i}$ has mean zero. Moreover $Q\left(v_{i}, v_{i}\right) \geqslant 0$ since $v_{i}$ has mean zero and $\partial \Omega_{i}$ is stable. Hence

$$
\begin{aligned}
Q(u, u) & =Q\left(u_{1}, u_{1}\right)+Q\left(u_{2}, u_{2}\right) \geqslant Q\left(c_{1}, c_{1}\right)+Q\left(c_{2}, c_{2}\right) \\
& =-\left(K+h^{2}\right)\left(t_{1}\right) c_{1}^{2} L\left(t_{1}\right)-\left(K+h^{2}\right)\left(t_{2}\right) c_{2}^{2} L\left(t_{2}\right) .
\end{aligned}
$$

Since $u$ has mean zero we have $c_{1} L\left(t_{1}\right)=-c_{2} L\left(t_{2}\right)$ and we conclude

$$
Q(u, u) \geqslant\left(-\frac{K+h^{2}}{L}\left(t_{1}\right)-\frac{K+h^{2}}{L}\left(t_{2}\right)\right) c_{1}^{2} L\left(t_{1}\right)^{2} \geqslant 0 .
$$

The last inequality by (1.7) holds. So we have proved that $\partial \Omega$ is stable.

Suppose now that $f(t)=f(-t)$ and that $t_{1}=-t_{2}$. Observe that $K(t)=K(-t)$, that $h(t)^{2}=h(-t)^{2}$, and that $L(t)=L(-t)$. So in this case inequalities (1.7) and (1.8) are equivalent. Moreover inequality (1.8) and Lemma 1.6 show that each component of $\partial \Omega$ is stable. From these observations the assertion on $\mathbb{S}^{1} \times\left[-t_{2}, t_{2}\right]$ follows easily.

On a complete Riemannian surface $M$ an isoperimetric domain $\Omega \subset M$ for area $A$ is a set enclosing area $A$ such that $\partial \Omega$ is smooth and has minimum length amongst the boundaries of smooth sets enclosing area $A$.

In a compact surface $M$ standard existence and regularity results from geometric measure theory imply that isoperimetric domains exist for any positive value less than or equal to the area of $M$, and that their boundaries are closed embedded stable curves. So a way to determine the isoperimetric domains is to classify the closed embedded curves with constant geodesic curvature or at least the stable ones.

For $A \in(0, A(M))$ we consider the function

$$
I(A)=\inf \{L(\partial B) ; B \text { is smooth and } A(B)=A\},
$$

which will be called the isoperimetric profile of $M([2])$. 
The usual way of proving existence of isoperimetric domains in a Riemannian surface $M$ for a given value $0<A<A(M)$ is to take a minimizing sequence of domains $\left\{\Omega_{n}\right\}_{n \in \mathbb{N}}$ with smooth boundary enclosing area $A$ such that

$$
\lim _{n \rightarrow \infty} L\left(\partial \Omega_{n}\right)=I(A)
$$

In general there is no convergence result for such sequences. However from standard Geometric Measure Theory ([10]) one can prove that each set in a minimizing sequence $\left\{\Omega_{n}\right\}_{n \in \mathbb{N}}$ can be decomposed as $\Omega_{n}=\Omega_{n}^{d} \cup \Omega_{n}^{c}$. The sequence $\Omega_{n}^{c}$ converges to some set $\Omega$ (which could be empty), and the sequence $\Omega_{n}^{d}$ diverges.

Lemma 1.8. Let $M$ be a Riemannian surface, $A \in(0, A(M))$, and let $\Omega_{n}$ be a minimizing sequence for area $A$. Then there is a (possibly empty) set $\Omega \subset M$ with $A(\Omega) \leqslant A$ with smooth boundary and a subsequence of $\Omega_{n}$, which will be denoted in the same way, such that $\Omega_{n}$ can be decomposed as $\Omega_{n}=\Omega_{n}^{c} \cup \Omega_{n}^{d}$, where $\Omega_{n}^{c}$ and $\Omega_{n}^{d}$ are union of connected components of $\Omega_{n}$. Moreover

(i) $\Omega_{n}^{c}$ converges to $\Omega$ locally as Cacciopoli sets,

(ii) $\Omega_{n}^{c}$ is relatively compact for each $n$ and the sequence $\left\{\Omega_{n}^{d}\right\}$ diverges,

(iii) If $L_{c}=\lim L\left(\partial \Omega_{n}^{c}\right)$ and $L_{d}=\lim L\left(\partial \Omega_{n}^{d}\right)$ then $L_{c}+L_{d}=L$.

(iv) $\partial \Omega$ is smooth, has constant geodesic curvature and it is stable.

\section{Planes.}

Let $M=\left(\mathbb{R}^{2}, d s^{2}\right)$ be a Riemannian plane, where $d s^{2}$ is a complete metric which is symmetric with respect to the usual Euclidean rotations in $\mathbb{R}^{2}$ around the origin. Removing the origin (the pole of the metric) from $M$ we obtain a Riemannian surface $N$ diffeomorphic to $\mathbb{S}^{1} \times I$, where $I=$ $(0, \infty)$. If $\theta \in \mathbb{S}^{1}$ and $t \in \mathbb{R}$ then the metric $d s^{2}$ restricted to $N$ is given by $d t^{2}+f(t)^{2} d \theta^{2}$, for some smooth function $f: I \rightarrow \mathbb{R}^{+}$.

The function $f(t)$ admits the following asymptotic expansion around $t=0$

$$
f(t)=t-\frac{K_{0}}{3 !} t^{3}+o\left(t^{4}\right)
$$

This follows from the Taylor type formula for the length $L$ of the geodesic circles at $L=0([7])$. In the above formula $K_{0}$ is the Gauss curvature at the pole and $o\left(t^{4}\right) / t^{4}$ is a bounded function around $t=0$. Such a formula implies 
that the derivatives up to third order of the function $f$ extend continuously to $t=0$ and that $f(0)=0, f^{\prime}(0)=1, f^{\prime \prime}(0)=0$ and $f^{\prime \prime \prime}(0)=-K_{0}$.

\subsection{Planes with decreasing curvature.}

In the conditions stated at the beginning of this section we assume now that the Gauss curvature $K(t)$ is a decreasing function of $t$. If $K(t)$ is smooth then $K^{\prime}(t) \leqslant 0$. This is equivalent to that the function $\left(f^{\prime}\right)^{2}-f f^{\prime \prime}$ is decreasing. If the surface is regular at $t=0$ then this function approaches 1 when $t$ goes to zero. Hence

$$
\left(f^{\prime}\right)^{2}-f f^{\prime \prime} \leqslant 1, \quad \text { for } \quad t>0,
$$

and the geodesic circles centered at the pole are stable by Lemma 1.6. Note that $\left(f^{\prime}\right)^{2}-f f^{\prime \prime}$ equals 1 precisely on some region of constant curvature around the pole.

The geometry of $M$ is described in next Lemma ([15, Lemma 3.2])

Lemma 2.1. For a surface $M$ in the above conditions one of the following possibilities holds

(i) $f^{\prime} \geqslant 0$ except possibly at some closed bounded or unbounded interval where $f^{\prime}$ vanishes.

(ii) There is $t_{1}>0$ such that $f^{\prime}(t)>0$ for $t<t_{1}$ and $f^{\prime}(t)<0$ for $t>t_{1}$.

(iii) There are $t_{1}, t_{2}$, with $0<t_{1}<t_{2}$, such that $f^{\prime}(t)>0$ for $t \in\left(0, t_{1}\right) \cup$ $\left(t_{2},+\infty\right)$ and $f^{\prime}(t)<0$ for $t \in\left(t_{1}, t_{2}\right)$.

Moreover in the first and third cases the area of $M$ is infinite. In the second one the area of $M$ is finite. If $\lim _{t \rightarrow+\infty} K(t)=K_{\infty}<0$ and the area of $M$ is infinite then the injectivity radius goes to $+\infty$ for any diverging sequence of points.

Proof. Since $K(t)$ is decreasing we have either

(a) $K(t)>0$ everywhere, or

(b) $K(t) \geqslant 0$ and $K(t)=0$ at some closed unbounded interval, or

(c) There exists a closed bounded interval $J$ such that $K(t)>0$ for $t<$ $\inf J, K(t)=0$ on $J$, and $K(t)<0$ for $t>\sup J$. 

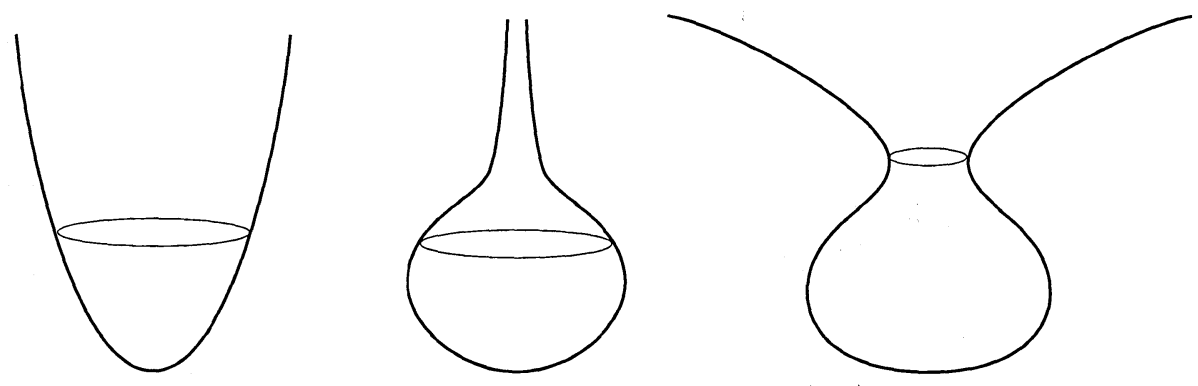

Figure 2: Planes with decreasing curvature.

Note that $f^{\prime}(t) \leqslant-C, C>0, t \rightarrow \infty$, is not possible since $f(t)>0$. As $f^{\prime}(0)=1$ and $\left(f^{\prime}\right)^{\prime}=-f K$, an elementary analysis of the possibilities gives (i), (ii) and (iii).

The area in cases (i) and (iii) is infinite since $f^{\prime}(t) \geqslant 0$ for $t$ large enough and so $f(t)>C$ for some $C>0$ and $t$ large enough.

In case (ii) we claim that there is $T>0$ such that $K(t)<0$ for $t>T$. Otherwise $K(t) \geqslant 0$ for $t$ large enough and so $f^{\prime}$ would be decreasing and less than or equal to some negative constant $-C$ for $t$ large enough, forcing $f$ to take negative values. This proves the claim. Taking $T$ large enough we may assume that $f^{\prime}(T)<0$. As the curvature is decreasing we have $-f^{\prime \prime} / f<-M$ for some $M>0$ in $(T,+\infty)$ and hence

$$
\int_{T}^{+\infty} f(\xi) d \xi<\frac{1}{M} \int_{T}^{+\infty} f^{\prime \prime}(\xi) d \xi=\frac{1}{M}\left(f^{\prime}(+\infty)-f^{\prime}(T)\right)
$$

If $f^{\prime}(+\infty)<0$ then $f$ would become negative at infinity. So $f^{\prime}(+\infty)=0$ and

$$
\int_{T}^{+\infty} f(\xi) d \xi<-\frac{f^{\prime}(T)}{M}<+\infty
$$

which implies that the area of $M$ is finite.

Assume now that $K_{\infty}<0$ and that the area of $M$ is infinite. Let us see that the injectivity radius goes to $+\infty$ for any diverging sequence of points. Consider a region $t \geqslant t_{0}$ where $f^{\prime \prime}>0$ and $f^{\prime}>0$. Let $p \in\left\{t \geqslant t_{0}\right\}$. We refer the reader to Chavel's book $([5])$ for definitions and properties of the cut locus $C(p)$. Assume there is $q \in\left\{t \geqslant t_{0}\right\}$ where the distance $d(p, C(p))$ is achieved. By Klingenberg's Lemma ([5]) there are two minimizing geodesics $\alpha, \beta:[0, L] \rightarrow M$ parameterized by arc-length such that $\alpha(0)=\beta(0)=p$, $\alpha(L)=\beta(L)=q$ and $\alpha^{\prime}(L)=-\beta^{\prime}(L)$. The points $p$ and $q$ cannot lie on 
the the same vertical geodesic $\theta=\theta_{0}$ since trivially the only minimizing geodesic joining $p$ and $q$ is precisely $\theta=\theta_{0}$. Parameterize $\alpha \cup(-\beta)$ so that $f(t) \sin \sigma=E>0$. From $(*)$

$$
\frac{d^{2} t}{d s^{2}}=\frac{f^{\prime}(t)}{f(t)} \sin ^{2} \sigma>0,
$$

and we conclude that $\alpha \cup(-\beta)$ has only minima of the $t$-coordinate. Equality $f(t) \sin \sigma=E$ implies that $\sin \sigma$ is a positive decreasing function of $t$ and so it has only one minimum of the $t$-coordinate. The point $p$ corresponds to the selfintersection of $\alpha \cup(-\beta)$ and the point $q$ is the point on $\alpha \cup(-\beta)$ where $t$ achieves its minimum value $t_{1} \geqslant t_{0}$. As $f(t)$ is increasing in $t \geqslant t_{0}$

$$
2 L \geqslant 2 \pi f\left(t_{0}\right) .
$$

For $p_{n}$ diverging, choose $t_{n} \in\left(t_{0}, t\left(p_{n}\right)\right.$ such that $t_{n} \rightarrow+\infty$ and $t\left(p_{n}\right)-t_{n} \rightarrow$ $+\infty$. Then the injectivity radius at $p$ is larger than or equal to

$$
\min \left\{t\left(p_{n}\right)-t_{n}, \pi f\left(t_{n}\right)\right\},
$$

which goes to $+\infty$ when $n \rightarrow+\infty$.

Next we classify the curves in $M$ having maxima and minima of the $t$-coordinate.

Lemma 2.2. Consider a rotationally symmetric surface with metric $d s^{2}=$ $d t^{2}+f(t)^{2} d t^{2}$ and a pole for $t=0$. Let $C \subset M$ be a curve with constant geodesic curvature $h$ in the above surface and assume that $\left.t\right|_{C}$ achieves local maxima and minima. Then $C$ is a nodoid, an unduloid, a geodesic circle around the pole, or a curve approaching the pole. In the last case the curve $C$ is a graph over $\theta$ with exactly one maximum for the $t$-coordinate and it meets the line $t=0$ orthogonally.

Proof. Parameterize $C$ by a solution $(\theta(s), t(s))$ to $(*)$ so that $t(0)=T$ is a maximum of the $t$-coordinate and $\sin \sigma(0)=1$. Let $E$ be the energy of the parameterized curve.

If $C$ is not a geodesic circle around the pole then $\left.t\right|_{C}$ has a strict maximum at $s=0$. If the minimum of $\left.t\right|_{C}$ is positive then $C$ is either an unduloid or a nodoid by Proposition 1.3. 
If $C$ approaches the pole then $E=0$. By the first integral (**) we have $f(T)-h \int_{0}^{T} f(\xi) d \xi=0$ and so $h>0$. Moreover

$$
\sin \sigma=\frac{h \int_{0}^{t} f(\xi) d \xi}{f(t)}
$$

and so $\sin \sigma>0$ and $C$ is a graph over $\theta$. When $t \rightarrow 0$ the above fraction goes to 0 by L'Hopital rule and so $C$ meets $t=0$ orthogonally.

Lemma 2.3. Consider a rotationally symmetric surface with metric $d s^{2}=$ $d t^{2}+f(t)^{2} d t^{2}$ and a pole for $t=0$.

(i) There are no closed embedded unduloids in regions where the function $\left(f^{\prime}\right)^{2}-f f^{\prime \prime} \leqslant 1$ but not identically 1 .

(ii) There are no closed embedded nodoids in regions where the Gauss curvature is decreasing or increasing and not constant.

(iii) There are no closed embedded curves touching a pole inside regions where the Gauss curvature is increasing or decreasing but not constant.

Proof. Take a curve with constant geodesic curvature and parameterize it so that $\sin \sigma=1$ at a maximum of $\left.t\right|_{C}$.

(i) If $C$ is an unduloid, we may assume that a minimum of $\left.t\right|_{C}$ is achieved at $\theta=0$, and that the first maximum of $\left.t\right|_{C}$ in the region $\{\theta>0\}$ lies over $\theta_{0}>0$. The curve $C$ yields a closed embedded curve in $M$ if and only if $\theta_{0}=\pi / k$, for some $k \in \mathbb{N}$. The function $\cos \sigma(\theta)$ is a positive solution of $(1.3)$ on $\left(0, \theta_{0}\right)$ which vanishes at $\theta=0$. Since $\left(f^{\prime}\right)^{2}-f f^{\prime \prime} \leqslant 1$ and $\not \equiv 1$, we can compare it with $\sin \theta$, the positive solution to $u^{\prime \prime}+u=0$ in $(0, \pi)$ which vanishes at $\theta=0$ using Lemma 1.5. We conclude that $\theta_{0}>\pi$. This proves (i).

(ii) Assume that $C$ is a nodoid. Translate it until a point with $\cos \sigma=1$ lies over $\theta=0$ and $C \subset\{\theta \geqslant 0\}$. We can suppose that $\sigma=0$ at this point. Let $\theta_{0}$ and $\theta_{1}>0$, be the projection over $\theta$ of the points with $\sigma=-\pi / 2$ and $\sigma=\pi / 2$, respectively. We obtain a closed embedded curve if and only if $\theta_{0}=\theta_{1}$. The pieces of $C$ corresponding to the $\sigma$-intervals $[-\pi / 2,0)$, $(0, \pi / 2]$ are graphs over $\theta$. The function $\cos \sigma$, restricted to each piece, gives two positive solutions to $(1.3)$ over $\left[0, \theta_{0}\right],\left[0, \theta_{1}\right]$, respectively. Observe that $\left.\cos \sigma\right|_{\theta=0}=1$ and that $(d \cos \sigma) /\left.d \theta\right|_{\theta=0}=-h f$. We can compare them using Lemma 1.5. If $K(t)$ is decreasing and not constant then $\left(f^{\prime}\right)^{2}-f f^{\prime \prime}$ 
is also and so $\theta_{0}<\theta_{1}$. If $K(t)$ is increasing and not constant then $\theta_{0}>\theta_{1}$. This proves (ii).

(iii) Assume now that $C$ approaches the pole. We know that $C$ is a graph over $\theta$ with one maximum for the $t$-coordinate and that $C$ is symmetric with respect to this maximum. Translate $C$ until it meets $t=0$ at $\theta=0$ and the maximum of $\left.t\right|_{C}$ lies over $\theta_{0}>0$. Then $C$ is smooth at the pole if and only if $\theta_{0}=\pi / 2$. We compare $\cos \sigma$ with $\cos \theta$, using Lemma 1.5 and the hypotheses on $\left(f^{\prime}\right)^{2}-f f^{\prime \prime}$. If $K(t)$ is decreasing and not constant then $\left(f^{\prime}\right)^{2}-f f^{\prime \prime} \leqslant 1$ and not identically 1 over $C$. It follows that $\theta_{0}>\pi / 2$. If $K(t)$ is increasing and not constant we have $\theta_{0}<\pi / 2$. In any case (iii) follows.

Theorem 2.4. The only connected closed embedded curves with constant geodesic curvature in a rotationally symmetric plane $M$ with decreasing curvature are the geodesic circles centered at the pole and possibly the boundaries of geodesic discs with constant Gauss curvature in $M$.

Proof. Let $C \subset M$ be a closed embedded curve with constant geodesic curvature. If $C$ is contained inside some region with constant Gauss curvature around the pole then $C$ is a geodesic circle. If $C$ is contained in a region with constant Gauss curvature which does not contain the pole then $C$ is of nodoid type and it bounds a disc with constant Gauss curvature. If $C$ is not contained in a region with constant Gauss curvature then it must be a circle of revolution by Lemmae 2.2 and 2.3 .

Remark 2.5. All the results in this section are still true if we consider a metric $d t^{2}+f(t)^{2} d \theta^{2}$ with $0<f^{\prime}(0)<1$, which is singular at the origin. However if the singularity comes from inequality $f^{\prime}(0)>1$ then there exist closed unduloids in the surface.

Remark 2.6. The only regularity hypotheses needed for $f$ are that $f$ is $C^{1}$ and piecewise $C^{2}$. Then the Gauss curvature $K$ is not continuous but it is decreasing if and only if $L^{2}\left(K+h^{2}\right)$ is decreasing. The version of Sturm's Comparison Theorem ([19]) that we need is satisfied with these regularity hypotheses.

Next we are going to prove that isoperimetric domains do exist on $M$. This has also been shown in [15]. Our proof is a new argument and show existence amongst domains with any number of connected components. The 
reader can find a related argument in the second part of Fiala's paper ([8]). First we state a result that we shall need in several sections of the paper.

Lemma 2.7. Let $\left\{D_{a}^{(1)}\right\},\left\{D_{a}^{(2)}\right\}, a \in\left(0, a_{0}\right)$, be smooth families of discs such that, for all a

(i) $A\left(D_{a}^{(1)}\right)=A\left(D_{a}^{(2)}\right)=a$,

(ii) $\partial D_{a}^{(1)}$ and $\partial D_{a}^{(2)}$ have constant geodesic curvature (not necessarily the same), and

(iii) $\int_{D_{a}^{(1)}} K d M \leqslant \int_{D_{a}^{(2)}} K d M$.

Then $L\left(\partial D_{a}^{(1)}\right) \geqslant L\left(\partial D_{a}^{(2)}\right)$ for all a. If inequality (iii) is strict then $L\left(\partial D_{a}^{(1)}\right)>L\left(\partial D_{a}^{(2)}\right)$.

Proof. Fix $a$ and $i=1,2$, and let $\varphi$ be the normal component of the variational field associated to the deformation $\partial D_{a}^{(i)}$. Then we have

$$
\frac{d L\left(\partial D_{a}^{(i)}\right)^{2}}{d a}=2 L\left(\partial D_{a}^{(i)}\right) \frac{\int_{\partial D_{a}^{(i)}} h\left(\partial D_{a}^{(i)}\right) \varphi}{\int_{\partial D_{a}^{(i)}} \varphi}=2 \int_{\partial D_{a}^{(i)}} h\left(\partial D_{a}^{(i)}\right)
$$

since $h\left(\partial D_{a}^{(i)}\right)$ is constant. By Gauss-Bonnet

$$
\frac{d L\left(\partial D_{a}^{(i)}\right)^{2}}{d a}=2\left(2 \pi-\int_{D_{a}^{(i)}} K d M\right) .
$$

Comparing the derivatives so obtained using (iii) and integrating from 0 to $a$ the Lemma follows.

Theorem 2.8. Let $M$ be a rotationally symmetric plane with a metric of curvature decreasing from the pole. Then isoperimetric domains exists on $M$ and they are bounded by geodesic circles around the pole.

Remark 2.9. For a characterization of isoperimetric domains in these surfaces we refer to the reader to [15, Theorem 3.1]. It turns out that isoperimetric domains are geodesic discs about the pole, annuli of revolution enclosing a least length circle of revolution and, in the case of finite area, the complements of geodesic discs around the pole. 
Proof. By Lemma 1.8 we only have to show that there is no area loss at infinity. This clearly cannot happen when the area of $M$ is finite. So we assume that $A(M)=\infty$. Take $A>0$ and a minimizing sequence $\Omega_{n}=$ $\Omega_{n}^{c} \cup \Omega_{n}^{d}$ for area $A$. The sequence $\Omega_{n}^{c}$ converges to a set $\Omega$ enclosing area $A_{c} \leqslant A$. The curve $\partial \Omega$ is smooth, it has constant geodesic curvature $h$ and it is stable. Let $K_{\infty}$ be the limit of the curvature at the end of $M$.

Case 2.10. $K_{\infty}<0$.

In this case $K$ is strictly negative near infinity. As the area of $M$ is infinite Lemma 2.1 shows that the injectivity radius of $M$ goes to infinity when $t \rightarrow \infty$.

Recall that for a disc $D$ with area $A$ and perimeter $L$ in a surface with Gauss curvature $K \leqslant K_{0}$ the following isoperimetric inequality holds ([8], [12])

$$
L^{2} \geqslant 4 \pi A-K_{0} A^{2} .
$$

If the surface is a plane and $K_{0}<0$ then this inequality is also valid for any domain $\Omega$. This follows since $\Omega$ is a disc $D \subset M$ with a finite number of holes removed. Clearly $L(\partial \Omega) \geqslant L(\partial D)$ and $A(D) \geqslant A(\Omega)$. As $K_{0}<0$ we have

$$
L(\partial \Omega)^{2} \geqslant L(\partial D)^{2} \geqslant 4 \pi A(D)-K_{0} A(D)^{2} \geqslant 4 \pi A(\Omega)-K_{0} A(\Omega)^{2} .
$$

This inequality is also valid for a domain with any number of connected components by inequality $\left(\sum_{i} a_{i}+\left(\sum_{i} b_{i}\right)^{2}\right)^{1 / 2}<\sum_{i}\left(a_{i}+b_{i}^{2}\right)^{1 / 2}$, for $a_{i}$, $b_{i}>0$.

Let $L_{d}=\lim _{n \rightarrow \infty} L\left(\partial \Omega_{n}^{d}\right)$ and $A_{d}=\lim _{n \rightarrow \infty} A\left(\Omega_{n}^{d}\right)$ be the limit length and area of the diverging sequence $\Omega_{n}^{d}$. Applying inequality (2.2) to each connected component of $\Omega_{n}^{d}$, summing up and passing to the limit we have

$$
L_{d}^{2} \geqslant 4 \pi A_{d}-K_{\infty} A_{d}^{2}
$$

If $K_{\infty}=-\infty$ then $A_{d}=0$. Otherwise, one would have $L_{d}=+\infty$, which is not possible since $L_{d}<+\infty$. In this case there is no loss of area. So let us assume from now on that $K_{\infty}$ is finite. Let us see that in fact equality holds in the above inequality. Consider a disc $D_{\infty}$ in a plane $M\left(K_{\infty}\right)$ of constant curvature $K_{\infty}$ such that $A\left(\Omega_{\infty}\right)=A_{d}$. Its perimeter equals $4 \pi A_{d}-$ $K_{\infty} A_{d}^{2}$. If the displayed above inequality is strict then $L\left(\partial D_{\infty}\right)<L_{d}$. Approximating $D_{\infty}$ by geodesic discs in $M$ centered in a diverging sequence of points with the same area as $\Omega_{n}^{d}$ we obtain a better minimizing sequence. This contradiction shows that $L\left(\partial D_{\infty}\right)=L_{d}$ 
Let us see now that $A_{c}, A_{d}$ cannot be positive at the same time. Denote by $h_{\infty}$ the geodesic curvature of $D_{\infty}$. We claim that

$$
h=h_{\infty},
$$

since otherwise we can find deformations $\Omega_{t},\left(D_{\infty}\right)_{t}$ of $\Omega$ in $M$ and of $D_{\infty}$ in $M\left(K_{\infty}\right)$ by parallel curves such that $A\left(\Omega_{t}\right)+A\left(\left(D_{\infty}\right)_{t}\right)=A_{c}+A_{d}$ and the sum of the boundary lengths of $\Omega_{t}$ and of $\left(\Omega_{\infty}\right)_{t}$ is less than $L_{c}+L_{d}$ for some $t$. Approximating the domain $\left(D_{\infty}\right)_{t}$ by geodesic discs in $M$ with the same area as $\Omega_{n}^{d}$ (this can be done since the injectivity radius of $M$ goes to infinity when we approach the end) we obtain a better solution to the isoperimetric problem. This contradiction shows that $h_{\infty}=h$.

The same argument implies that if we make a variation of $\Omega \cup D_{\infty}$ by parallels keeping the area enclosed constant then the second derivative of perimeter is nonnegative. Hence if we consider the function

$$
u= \begin{cases}-L_{c}, & \partial D_{\infty}, \\ L_{d}, & \partial \Omega,\end{cases}
$$

then the index form (1.4) applied to $u$ should give a nonnegative value but

$$
\begin{aligned}
I(u) & =-\int_{\partial D_{\infty}}\left(K_{\infty}+h^{2}\right) L_{c}^{2}-\int_{\partial \Omega}\left(K+h^{2}\right) L_{d}^{2} \\
& \leqslant-\int_{\partial D_{\infty}}\left(K_{\infty}+h^{2}\right) L_{c}^{2}-\int_{\partial \Omega}\left(K_{\infty}+h^{2}\right) L_{d}^{2},
\end{aligned}
$$

since $\left.K\right|_{\partial \Omega} \geqslant K_{\infty}$. As $K_{\infty}+h^{2}$ is positive for geodesic circles in $M\left(K_{\infty}\right)$ the last quantity is negative. This gives us a contradiction that shows that either $A_{d}=0$ or $A_{c}=0$. If $A_{d}=0$ the proof is complete. If $A_{c}=0$ then a geodesic disc centered at the pole is better than a disc at infinity since the curvature of the former is larger than the one of the last by Lemma 2.7.

Case 2.11. $K_{\infty}=0$.

In this case the curvature of $M$ is nonnegative, $\int_{M} K \leqslant 2 \pi$ by CohnVossen inequality and, since $f^{\prime \prime}<0$, it follows that $-1<\int_{0}^{t} f^{\prime \prime}(\xi) d \xi<0$, and so we have $0 \leqslant f^{\prime}<1$.

We consider the conformal field $X=f(t) \partial_{t}$ and the associated oneparameter group $\varphi_{t}$ of diffeomorphisms. As $f^{\prime} \geqslant 0$ equation (1.2) and the first variation formula for the area imply that $\varphi_{t}$ increases area. Let us see that $\varphi_{t}$ also increases length of curves. Consider an embedded curve $C \subset M$. 
Let $v$ be a unit vector tangent at $p \in C$. Let $Z$ be a vector field around $p$ that coincides with $d \varphi_{s}(v)$ for $s$ small and such that $[X, Z]=0$. Then

$$
\left.\frac{d}{d s}\right|_{s=0}\left|d \varphi_{s}(v)\right|=\left\langle D_{X_{p}} Z, v\right\rangle=\left\langle D_{Z_{p}} X, v\right\rangle+\langle[X, Z](p), v\rangle=f^{\prime}|v|=f^{\prime} \geqslant 0 .
$$

Integrating on $s$ it follows that $\left|\varphi_{s}(v)\right| \geqslant|v|$ when $s>0$ and, using the area formula, we deduce that $L\left(\varphi_{s}(C)\right) \geqslant L(C)$ when $s>0$.

We can assume that each set $\Omega_{n}$ in the minimizing sequence is a union of discs. Observe that each connected component of $\Omega_{n}$ is a disc $D_{m}$ with a finite number of holes removed, and that there are at most a countable number of components. Since two different discs $D_{m}$ are either disjoint or one of them lies inside the other, the set $\bigcup_{m} D_{m}$ is a union of discs. We claim that $\bigcup_{m} D_{m}$ has finite area: otherwise there is a subsequence $D_{m_{k}}$ such that $\partial D_{m_{k}}$ diverges. The integral version of the isoperimetric inequality shows that a diverging sequence of disjoint discs has area controlled by the perimeter, which is bounded by the one of $\Omega_{n}$. So we may assume that $D_{m_{k}} \subset D_{m_{k+1}}$ for all $k$. But then $L\left(\partial D_{m_{k}}\right)$ is uniformly bounded below by some positive constant, and we get a contradiction since $\sum_{k} L\left(\partial D_{m_{k}}\right) \leqslant L\left(\partial \Omega_{n}\right)$, which proves the claim. Hence $\bigcup_{m} D_{m}$ has finite area larger than the one of $\Omega_{n}$. On the other hand the perimeter of $\bigcup_{m} D_{m}$ is less than or equal to the one of $\Omega_{n}$. Applying the one-parameter group of diffeomorphisms $\left\{\varphi_{t}\right\}$ associated to the conformal field $X$ we decrease the area and the perimeter of $\bigcup_{m} D_{m}$ to obtain a family of discs enclosing area $A$ and with perimeter less than or equal to the one of $\Omega_{n}$. We denote this minimizing sequence in the same way and apply Lemma 1.8 to get a limit set $\Omega$.

Since $\partial \Omega$ has constant geodesic curvature, Theorem 2.4 implies that $\partial \Omega$ is the union of circles of revolution and the boundaries of geodesic discs with constant curvature. If $\partial \Omega$ is not connected then using a locally constant test function with mean zero taking into account $K+h^{2} \geqslant 0$, we deduce that $\partial \Omega$ is the union of circles of revolution with $h=0$ in a flat region. As $f^{\prime} \geqslant 0$, a geodesic disc around the pole with the same area has less perimeter. If $\Omega$ is connected then $\Omega$ can be replaced by a geodesic disc centered at the pole by Lemma 2.7 .

The isoperimetric inequality for disc type domains in surfaces, the divergence of the sequence $\left\{\Omega_{n}^{d}\right\}$ and the fact that the positive Gauss curvature goes uniformly to zero outside of compact subsets implies

$$
L_{d}^{2} \geqslant 4 \pi A_{d}
$$


Consider now the geodesic circle $C$ centered at the pole enclosing area $A$. Let $L$ be the length of $\partial C$. We have

$$
\left(L_{c}+L_{d}\right)^{2} \geqslant L_{c}^{2}+L_{d}^{2} \geqslant 4 \pi\left(\pi f^{2}\left(A_{c}\right)+A_{d}\right),
$$

where $f(A)$ denotes the composition $f(t(A))$, for $t(A)$ the inverse function of the area of the geodesic circles of the radius $t$ around the pole. We claim that the following inequality holds

$$
\pi f^{2}\left(A_{c}\right)+A_{d}>\pi f^{2}(A), \quad \text { whenever } A=A_{c}+A_{d}, \quad A_{d}>0 .
$$

Indeed this inequality becomes an equality if $A_{d}=0$. Fixing $A_{c}$ and thinking about the two terms in the above inequality as functions of $A_{d}$ we easily see that the derivative of $\pi f^{2}\left(A_{c}+A_{d}\right)$ with respect to $A_{d}$ equals $f^{\prime}$ (the derivative of $f$ with respect to $t$ ), which is less than one. This proves the strict inequality and the claim. Hence we have

$$
\left(L_{c}+L_{d}\right)^{2} \geqslant L^{2}
$$

and equality implies that $L_{d}=0$ and so $A_{d}=0$. We conclude that strict inequality holds in the above displayed inequality. So if $A_{d}>0$ the geodesic circle $C$ encloses a better solution. This is clearly not possible. So $A_{d}=0$ and we find our reasoning.

Finally we show how one can reconstruct the surface from its isoperimetric profile in the convex case

Theorem 2.12. Let $I:(0,+\infty) \rightarrow \mathbb{R}$ be a smooth function such that $\left(I^{2}\right)^{\prime}$ is a nonnegative, decreasing, convex function such that $I^{2}(a) \rightarrow 0$, $\left(d I^{2} / d a\right)(a) \rightarrow 4 \pi$ when $a \rightarrow 0$.

Then there is a complete plane of revolution with a smooth metric such that $I(a)$ is the isoperimetric profile of $M$. Moreover, the Gauss curvature of $M$ is a nonnegative decreasing function.

Proof. Let $F(t)$ be the solution to

$$
F^{\prime \prime}(t)=\frac{d I^{2}}{d a}(F(t))
$$

with initial conditions $F(0)=F^{\prime}(0)=0$. As $F^{\prime \prime}(t) \geqslant 0$ and $F^{\prime \prime}(0)=2 \pi>0$ we have $F^{\prime}(t)>0$ for $t>0$. Since the function $d I^{2} / d a$ is decreasing we obtain $F^{\prime}(t) \leqslant 2 \pi$ and so $F(t)$ is defined for all $t$. We consider the function

$$
f(t)=\frac{F^{\prime}(t)}{2 \pi} .
$$


Then we have $F(t)=2 \pi \int_{0}^{t} f(\xi) d \xi$. The metric of revolution $d t^{2}+f(t)^{2} d \theta^{2}$ is complete, regular at the pole as $f^{\prime}(0)=F^{\prime \prime}(0) /(2 \pi)=1$. Moreover

$$
K(t)=-\frac{f^{\prime \prime}(t)}{f(t)}=-\frac{F^{\prime \prime \prime}(t)}{F(t)}=-2 \frac{d^{2} I^{2}}{d a^{2}} .
$$

Since $d I^{2} / d a$ is decreasing and convex we deduce that $K(t)$ is nonnegative and decreasing. The isoperimetric domains in $M$ are the geodesic discs centered at the pole. Let $L(a)$ be the isoperimetric profile of $M$. We have $a=F(t)$ and $d L^{2} / d a=2 L h=2 f^{\prime}(t)=2 F^{\prime \prime}(t)=d I^{2} / d a$. As $L^{2}(0)=$ $I^{2}(0)=0$ we have $L(a)=I(a)$.

\subsection{Planes with increasing curvature.}

We assume in this section that the Gauss curvature $K(t)$ is an increasing function of $t$. If $K(t)$ is smooth then $K^{\prime}(t) \geqslant 0$. We know that the function $\left(f^{\prime}\right)^{2}-f f^{\prime \prime}=(2 \pi)^{-2} L^{2}\left(K+h^{2}\right)(t)$ is also increasing. If $M$ is regular at the pole then $\left(f^{\prime}\right)^{2}-f f^{\prime \prime}$ approaches 1 when $t$ goes to zero and so

$$
\left(f^{\prime}\right)^{2}-f f^{\prime \prime} \geqslant 1, \quad \text { for } \quad t>0 .
$$

Note that $\left(f^{\prime}\right)^{2}-f f^{\prime \prime}$ equals 1 precisely on some region of constant curvature around the pole. Hence the geodesic circles centered at the pole and contained in this region are stable and the ones in the complementary region are unstable by Lemma 1.6.

Since $K(t)$ is increasing and $M$ is complete, there exists the limit $K_{\infty}=$ $\lim _{t \rightarrow+\infty} K(t)$ and it is nonpositive. So $K(t) \leqslant 0$ everywhere. As $f^{\prime}(0)=1$ and $f^{\prime \prime}(t) \geqslant 0$ we have $f^{\prime}(t) \geqslant 1$ for all $t$ and $f(t)$ is a strictly increasing function. The injectivity radius goes to $+\infty$ when we approach the end as in Lemma 2.1.

We shall need the following Lemma

Lemma 2.13. Consider a rotationally symmetric surface with metric $d t^{2}+$ $f(t)^{2} d \theta^{2}$. Let $C$ be a closed embedded unduloid such that $\left(f^{\prime}\right)^{2}-f f^{\prime \prime} \geqslant 1$ and $\left(f^{\prime}\right)^{2}-f f^{\prime \prime}$ is not identically 1 over $C$. Then $C$ is unstable.

Proof. By Lemma 2.2 the $\theta$-distance between two consecutive maxima or minima of $\left.t\right|_{C}$ is less than $2 \pi$. Hence we need at least two pieces between adjacent maxima or minima of the $t$-coordinate to obtain a closed embedded curve. In this case the restriction of the Jacobi field $u=f(t) \cos \sigma$ to the 
curve has at least four nodal domains. Courant's Nodal Domain ([4]) then shows that the Jacobi operator has at least three negative eigenvalues and so the curve is unstable.

The result characterizing the stable embedded curves in such a plane is

Theorem 2.14. Let $M$ be a rotationally symmetric plane with increasing curvature. Then there are no stable curves embedded in $M$, except possibly the boundaries of geodesic discs with constant curvature. If the Gauss curvature is strictly increasing then there are no stable embedded curves in $M$.

Proof. By Lemma 2.2 an embedded connected curve in $M$ must be a nodoid, an unduloid, a curve touching the pole or a circle of revolution. By equation (2.3) we have $\left(\left(f^{\prime}\right)^{2}-f f^{\prime \prime}\right)(t) \geqslant 1$ and equality holds at some geodesic disc of radius $t_{0}$ around the pole with constant Gauss curvature. Of course $t_{0}$ can be either 0 or $+\infty$. Any curve with constant geodesic curvature contained in $t \leqslant t_{0}$ is the boundary of a geodesic disc with constant Gauss curvature.

Curves approaching the pole and touching $t>t_{0}$ cannot exist by Lemma 2.2. Unduloids touching $t>t_{0}$ are unstable by Lemma 2.13. Nodoids touching $t>t_{0}$ exist if they are contained inside a region with constant Gauss curvature and they are the boundaries of geodesic discs with constant Gauss curvature by Lemma 2.2. Circles of revolution are stable if and only if they are contained in $t \leqslant t_{0}$ by Lemma 1.6. We conclude that the only connected stable embedded curves are the boundaries of geodesic discs with constant curvature in $M$. Since these curves have negative first eigenvalue for the Jacobi operator $J$ we conclude that a stable curve has to be connected.

When the Gauss curvature is strictly increasing the only embedded curves with constant geodesic curvature are circles of revolution, which are not stable.

Remark 2.15. As in the previous section, the above result follows if $f$ is merely $C^{1}$ and piecewise $C^{2}$. It remains valid also if metric singularities of the type $f^{\prime}(0)>1$ are allowed.

Let us see now that isoperimetric domains can never exist on $M$

Theorem 2.16. Let $M$ be a rotationally symmetric plane of increasing curvature. Let $K_{\infty}=\sup _{M} K$. The isoperimetric domains in $M$ are geodesic 
discs with constant curvature $K_{\infty}$. If this region is void then isoperimetric domains do not exist on $M$.

Moreover, the isoperimetric profile of $M$ is given by

$$
I(a)^{2}=4 \pi a-K_{\infty} a^{2} .
$$

Proof. Fix $A \in(0, A(M))$, and let $\left\{\Omega_{n}\right\}_{n \in \mathbb{N}}$ be a minimizing sequence for area $A$. By Lemma 1.8 we have $\Omega_{n}=\Omega_{n}^{c} \cup \Omega_{n}^{d}$, where $\Omega_{n}^{c}$ is convergent to some domain $\Omega$ and $\Omega_{n}^{d}$ is divergent. Moreover $\partial \Omega$ is stable and so it is a geodesic circle in a region of constant curvature around the pole. We conclude that $\Omega$ is a geodesic disc with constant Gauss curvature $K_{0}$ and we have

$$
L(\partial \Omega)^{2}=4 \pi A(\Omega)-K_{0} A(\Omega)^{2} .
$$

On the other hand, for the divergent part of the sequence $\Omega_{n}^{d}$ we have

$$
L_{d}^{2}=4 \pi A_{d}-K_{\infty} A_{d}^{2}
$$

It is clear that the perimeter of a disc in $M\left(K_{\infty}\right)$ enclosing area $A$ is less than or equal to $L(\partial \Omega)+L_{d}$ (equality holds only if $L(\partial \Omega)=0$ or $L_{d}=0$ ). If the region where $K=K_{\infty}$ is non void then a geodesic disc of area $A$ inside this region is an isoperimetric domain.

\section{Spheres.}

In this section we consider a rotationally symmetric sphere $M$. The Killing field vanishes at two points. Removing them we obtain a Riemannian manifold isometric to $\mathbb{S}^{1} \times\left(0, t_{0}\right)$ with metric $d s^{2}=d t^{2}+f(t)^{2} d \theta^{2}$. The point corresponding to $t=0$ will be called the south pole, and the one corresponding to $t=t_{0}$ the north pole. The curves $\mathbb{S}^{1} \times\{t\}$ will be called parallels and the ones with $\theta$-coordinate constant meridians. The equator is the curve $\mathbb{S}^{1} \times\left\{t_{0} / 2\right\}$. We shall impose to this surface to have an equatorial symmetry with respect to $t=t_{0} / 2$, that is

$$
f(t)=f\left(t_{0}-t\right)
$$

Since $M$ is regular $f(t)$, and their derivatives up to third order, extend to $t=0$ and to $t=t_{0}$ and we have $f(0)=f\left(t_{0}\right)=0, f^{\prime \prime}(0)=f^{\prime \prime}\left(t_{0}\right)=0$, $f^{\prime}(0)=1, f^{\prime}\left(t_{0}\right)=-1, f^{\prime \prime \prime}(0)=-K_{s}, f^{\prime \prime \prime}\left(t_{0}\right)=K_{n}$, where $K_{s}=K_{n}$ are the values of the Gauss curvature at $t=0$ and $t=t_{0}$, respectively. 
We remark that there are no rotationally symmetric metrics with strictly monotone curvature in a sphere. This follows from an old argument by Kazdan and Warner ([16]), integrating the derivative of $\left(f^{\prime}\right)^{2}-f f^{\prime \prime}$ between 0 and $t_{0}$, or by the arguments in [15].

\subsection{Spheres with curvature increasing from the equator.}

In this subsection we assume that the Gauss curvature $K(t)$ is decreasing for $t<t_{0} / 2$ and so increasing for $t>t_{0} / 2$.

The function $\left(f^{\prime}\right)^{2}-f f^{\prime \prime}$ takes the value 1 at $t=0, t=t_{0}$, it decreases from 0 up to $t_{0} / 2$ and it increases from $t_{0} / 2$ up to $t_{0}$. So

$$
\left(f^{\prime}\right)^{2}-f f^{\prime \prime} \leqslant 1, \quad \text { on } \quad\left(0, t_{0}\right) .
$$

Equality holds on regions with constant Gauss curvature around both poles. Lemma 1.6 and the above inequality show that any circle of revolution is stable.

The geometric behavior of $M$ follows next Lemma. See Figure 3 .

Lemma 3.1. The Gauss curvature $K(t)$ is positive around the poles and either nonnegative everywhere or negative on a symmetric annulus around the equator.

(i) If $K(t) \geqslant 0$ everywhere then $f^{\prime}(t) \geqslant 0$ for $t \in\left(0, t_{0} / 2\right]$ and $f^{\prime}(t)=0$ precisely at some closed interval containing $t_{0} / 2$.

(ii) If $K(t)$ changes its sign then there is $t_{1} \in\left(0, t_{0} / 2\right)$ such that $f^{\prime}(t)>0$ for $t \in\left(0, t_{1}\right)$ and $f^{\prime}(t)<0$ for $t \in\left(t_{1}, t_{0} / 2\right)$.

Proof. The Gauss curvature is positive somewhere by Gauss-Bonnet. It achieves its maxima at the poles and decreases up to the equator, so it is everywhere nonnegative and it vanishes around the equator or it becomes negative around the equator.

Since $K(t)$ is decreasing in $\left(0, t_{0} / 2\right]$ and $K(0)>0$ we have that either

(a) $K(t)>0$ for $t \in\left(0, t_{0} / 2\right]$, or

(b) $K(t) \geqslant 0$ for $t \in\left(0, t_{0} / 2\right]$ and $K(t)=0$ at some closed interval in $\left(0, t_{0} / 2\right]$ containing $t_{0} / 2$, or

(c) there is a closed interval $J \subset\left(0, t_{0} / 2\right)$ such that $K(t)>0$ for $t<\inf J$, $\left.K\right|_{J}=0$ and $K(t)<0$ for $t>\sup J$. 

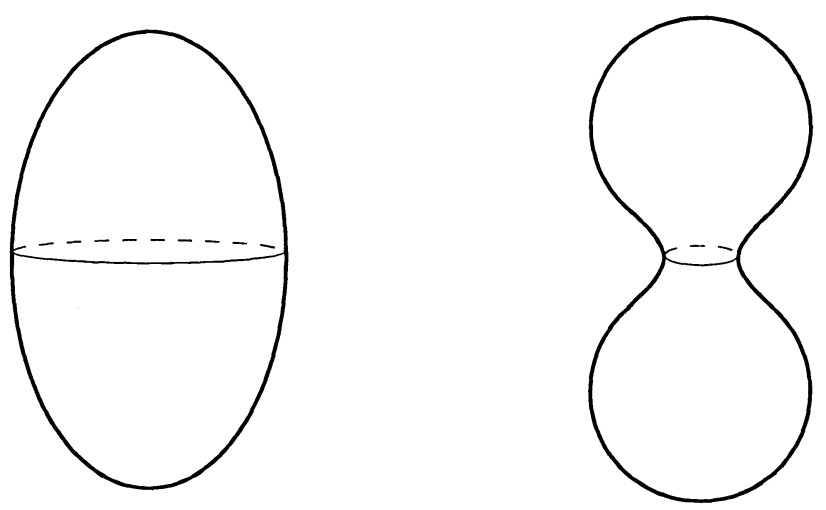

Figure 3: A convex sphere and a sphere with points of negative curvature.

As $\left(f^{\prime}\right)^{\prime}=-f K, f^{\prime}(0)=1$ and $f^{\prime}\left(t_{0} / 2\right)=0$ we obtain (ii) from (a) and (b) and we obtain (iii) from (c).

In these spheres of revolution there exist closed embedded nodoids. This will be shown for convex spheres

Lemma 3.2. Consider the surface $\mathbb{S}^{1} \times\left(0, t_{0}\right)$ with the Riemannian metric $d s^{2}=d t^{2}+f(t)^{2} d \theta^{2}$ such that $f\left(t-t_{0}\right)=f(t)$. Assume that $f^{\prime}(t) \geqslant 0$ for $t<t_{0} / 2$ and that $f^{\prime}(t) \leqslant 0$ for $t>t_{0} / 2$, with $f^{\prime}=0$ precisely at some interval containing $t_{0} / 2$.

Then there is a family of embedded nodoids $\left\{C_{T}\right\}$, for $T>t_{0} / 2$, which are symmetric with respect to $t=t_{0} / 2$. Moreover the geodesic curvature $h(T)$ is a positive strictly decreasing function of $T$.

Proof. Fix $T \in\left(t_{0} / 2, t_{0}\right)$ and define

$$
h(T)=f(T)\left(\int_{t_{0} / 2}^{T} f(\xi) d \xi\right)^{-1}
$$

Consider the solution $\theta, t, \sigma$, to $(*)$ with $h=h(T)>0$ and initial conditions $0, T, \pi / 2$. Evaluating the first integral $(* *)$ at $T$ and computing $f(T)$ from the definition of $h(T)$ we obtain for the energy the value

$$
E_{T}=-h(T) \int_{0}^{t_{0} / 2} f(\xi) d \xi
$$


and from $(* *)$

$$
\sin \sigma=\frac{h(T) \int_{t_{0} / 2}^{t} f(\xi) d \xi}{f(t)}
$$

So $\sin \sigma>0$ for $t>t_{0} / 2$ and $\sin \sigma=0$ at $t=t_{0} / 2$.

Since $h(T)>0$ and $\sin \sigma>0$ in $t>t_{0} / 2$ it follows from the third of equations $(*)$ that $d \sigma / d s \geqslant h>0$ whenever the curve stays inside the region $t>t_{0} / 2$. This implies that the solution hits the equator and that it meets it orthogonally. Reflecting with respect to the equator and again with respect to $\theta=0$ we obtain a closed embedded curve with constant geodesic curvature that we shall denote by $C_{T}$. The derivative of $h(T)$ with respect to $T$ equals

$$
\left(f^{\prime}(T) \int_{t_{0} / 2}^{T} f(\xi) d \xi-f(T)^{2}\right)\left(\int_{t_{0} / 2}^{T} f(\xi) d \xi\right)^{-2}
$$

which is strictly negative since $f^{\prime}(t) \leqslant 0$.

Theorem 3.3. Let $M$ be a rotationally symmetric sphere whose Gauss curvature is a decreasing function of the distance from the poles. Then the only connected closed embedded curves in $M$ with constant geodesic curvature are parallels, meridians, nodoids symmetric with respect to the equator which are graphs over the equator, and the boundaries of geodesic discs with constant Gauss curvature.

Moreover, the only stable ones are the parallels and the boundaries of geodesic discs with constant Gauss curvature.

Proof. Let $C \subset M$ be a closed embedded curve with constant geodesic curvature $h$.

If $C$ meets the south pole then we parameterize $C$ by a solution of $(*)$ with $E=0$. If, in addition, $C$ touches the north pole then $f\left(t_{0}\right)=0$ and (**) shows that $h=0$. Again by $(* *)$ we have $\sin \sigma=0$ and $C$ is a meridian.

We assume from now on that $C$ is not a meridian and hence that it does not touch both poles. By reflecting it with respect to the equator if necessary we can suppose that $C$ stays away from the north pole. If $C$ is contained in a region of constant curvature around a pole then $C$ is the boundary of a geodesic disc with constant Gauss curvature. Henceforth we assume that $C$ is not contained in a region of constant Gauss curvature around the poles.

As $\left(f^{\prime}\right)^{2}-f f^{\prime \prime} \leqslant 1$ and $\not \equiv 1$ Lemma 2.2 imply that $C$ is neither an unduloid nor a curve approaching the pole. 
If $C$ is a nodoid then we conclude by Lemma 2.3 and by next Lemma 3.4 that $C$ is either the boundary of a geodesic disc with constant Gauss curvature or a unstable nodoid symmetric with respect to the equator.

Finally circles of revolution are stable by Lemma 1.6.

Lemma 3.4. Consider the surface $\mathbb{S}^{1} \times\left(0, t_{0}\right)$ with the Riemannian metric $d s^{2}=d t^{2}+f(t)^{2} d \theta^{2}$ such that $f\left(t-t_{0}\right)=f(t)$. Assume that $K(t)$ is monotone for $t \in\left(t_{0} / 2, t_{0}\right)$. Let $C \subset \mathbb{S}^{1} \times\left(0, t_{0}\right)$ be a nodoid type curve which is not contained inside a region with constant Gauss curvature. Then we have

(i) The curve $C$ is embedded if and only if it is symmetric with respect to $t=t_{0} / 2$.

(ii) If $K(t)$ is increasing for $t \in\left(t_{0} / 2, t_{0}\right)$ and $C$ is embedded then $C$ is unstable.

Proof. First we prove (i). Obviously if $C$ is symmetric then it is embedded. Let $T=\left.\max t\right|_{C}, S=\left.\min t\right|_{C}$. We parameterize $C$ by a solution $(\theta, t)$ to (*) with initial conditions $0, T, \pi / 2$. Let $E$ be the energy and $h$ the geodesic curvature. As $C$ is a nodoid we have $E h<0(E h \geqslant 0$ implies graph over $\theta$ by $(* *))$. Let us assume that $E<0$ and $h>0$. The other possibility $E>0$, $h<0$ is reduced to the first one by traversing the curve in the opposite sense.

Since $C$ is embedded it cannot be contained in a region where the curvature is strictly monotone by Lemma 2.3. So $S<t_{0} / 2<T$. Moreover

$$
f(T)-h \int_{0}^{T} f(\xi) d \xi=E, \quad-f(S)-h \int_{0}^{S} f(\xi) d \xi=E
$$

If $E=-h \int_{0}^{t_{0} / 2} f(\xi) d \xi$ then $C$ meets the equator orthogonally and it is symmetric with respect to $t=t_{0} / 2$. So let us assume $E \neq-h \int_{0}^{t_{0} / 2} f(\xi) d \xi$.

If we reflect $C$ with respect to $t=t_{0} / 2$ we obtain a new curve $\widetilde{C}$ with the same geodesic curvature $h$ that can be parameterized by a solution $\tilde{\theta}, \tilde{t}$, $\widetilde{\sigma}$ to $(*)$ with initial conditions $0, t_{0}-S, \pi / 2$. The energy $\widetilde{E}$ of this solution is given by

$$
\widetilde{E}=f\left(t_{0}-S\right)-h \int_{0}^{t_{0}-S} f(\xi) d \xi
$$


and an easy computation using the symmetry $f\left(t_{0}-t\right)=f(t)$ yields

$$
\left(E+h \int_{0}^{t_{0} / 2} f(\xi) d \xi\right)+\left(\widetilde{E}+h \int_{0}^{t_{0} / 2} f(\xi) d \xi\right)=0 .
$$

So replacing $C$ by $\widetilde{C}$ if necessary we may assume that

$$
E>-h \int_{0}^{t_{0} / 2} f(\xi) d \xi
$$

From (3.1) and (**) we have $\sin \sigma>0$ when $t \geqslant t_{0} / 2$ and we obtain that $C \cap\left\{t>t_{0} / 2\right\}$ is a graph over $\theta$.

Claim. For $t \in\left(t_{0}-T, t_{0} / 2\right)$ we have $-f(t)-h \int_{0}^{t} f(\xi) d \xi<E$ and so $S<t_{0}-T$.

In order to prove the claim fix $t \in\left(t_{0}-T, t_{0} / 2\right)$. We have

$$
\begin{aligned}
-f(t)-h \int_{0}^{t} f(\xi) d \xi & =-f\left(t_{0}-t\right)+h \int_{0}^{t_{0}-t} f(\xi) d \xi-2 h \int_{0}^{t_{0} / 2} f(\xi) d \xi \\
& <-f\left(t_{0}-t\right) \sin \sigma+h \int_{0}^{t_{0}-t} f(\xi) d \xi-2 h \int_{0}^{t_{0} / 2} f(\xi) d \xi \\
& =E-2 E-2 h \int_{0}^{t_{0} / 2} f(\xi) d \xi<E .
\end{aligned}
$$

In the first line we have used the symmetry $f\left(t_{0}-t\right)=f(t)$, we have added and subtract $h \int_{0}^{t_{0}-t} f(\xi) d \xi$ and taken into account that $\int_{0}^{t_{0}-t} f(\xi) d \xi=$ $\int_{t}^{t_{0}} f(\xi) d \xi$ and that $\int_{0}^{t_{0}} f(\xi) d \xi=2 \int_{0}^{t_{0} / 2} f(\xi) d \xi$. In the second one we have used inequality $\sin \sigma<1$ and equality $\int_{0}^{t_{0}} f(\xi) d \xi=2 \int_{0}^{t_{0} / 2} f(\xi) d \xi$. In the last one we have added and subtract $E$ and applied (3.1). Inequality $S<t_{0}-T$ follows trivially and proves the claim.

Let us see that $C$ lies below $\widetilde{C}$ in the region $t>t_{0} / 2$, see Figure 4 .

If both curves meet at some point we have $t=\widetilde{t}$ and

$$
f(t)(\sin \sigma-\sin \widetilde{\sigma})=E-\widetilde{E} .
$$

Evaluating at $t_{0} / 2$ we conclude, as $\sin \sigma>0, \sin \widetilde{\sigma}<0$, that $E-\widetilde{E}>0$. So $\sin \sigma>\sin \widetilde{\sigma}$ at any point where both curves cross. Initially $\widetilde{t}>t$. Let $\theta_{0}<0$ be the smaller value where $\tilde{t}=t$. At this value both $\tilde{t}$ and $t$ are graphs over $\theta$, so we have $(d t / d \theta)\left(\theta_{0}\right) \geqslant(d \widetilde{t} / d \theta)\left(\theta_{0}\right)$, which implies $\cot \sigma\left(\theta_{0}\right) \geqslant \cot \widetilde{\sigma}\left(\theta_{0}\right)$ and so $\sin \sigma\left(\theta_{0}\right) \leqslant \sin \widetilde{\sigma}\left(\theta_{0}\right)$, which contradicts the above. 


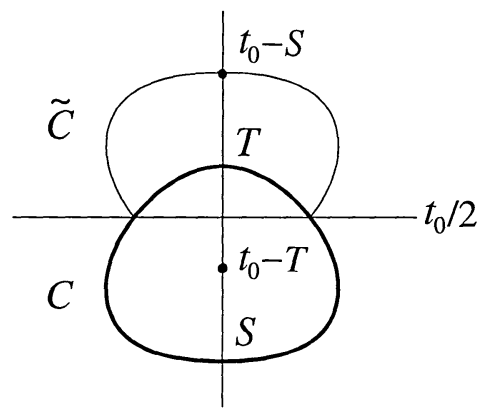

Figure 4.

Get two points $\left(\theta_{0}, t_{1}\right),\left(\theta_{0}, t_{2}\right)$ in $C$ with the same $\theta_{0}$ and $t_{1}<t_{2}$. Assume that $K(t)$ is increasing in $\left(0, t_{0} / 2\right)$. If $t_{2}<t_{0} / 2$ then $\left(\left(f^{\prime}\right)^{2}-\right.$ $\left.f f^{\prime \prime}\right)\left(t_{1}\right) \leqslant\left(\left(f^{\prime}\right)^{2}-f f^{\prime \prime}\right)\left(t_{2}\right)$. If $t_{2}>t_{0} / 2$ then the property proved in the previous paragraph shows that $t_{0}-t_{2}>t_{1}$. As $t_{0}-t_{2}<t_{0} / 2$ and the function $\left(f^{\prime}\right)^{2}-f f^{\prime \prime}$ is symmetric with respect to $t=t_{0} / 2$ we conclude that $\left(\left(f^{\prime}\right)^{2}-f f^{\prime \prime}\right)\left(t_{1}\right) \leqslant\left(\left(f^{\prime}\right)^{2}-f f^{\prime \prime}\right)\left(t_{2}\right)$. Moreover there are at least two such points where the inequality is strict since $C$ is not contained in a region with constant curvature. If $K(t)$ is decreasing in the interval $\left(0, t_{0} / 2\right)$ reverse inequalities are obtained.

Hence we can apply the same argument as in the proof of Lemma 2.3(ii) to show that $C$ cannot be closed.

To see (ii) we consider the test function

$$
u(s)=\left(f^{\prime}(t)-h f(t) \sin \sigma\right)(s)=\frac{d^{2}}{d s^{2}} \int_{0}^{t(s)} f(\xi) d \xi,
$$

which has mean zero when integrated over $C$. Since $C$ is symmetric by (i) we see that $u$ vanishes over the equator. At any point where $\sin \sigma= \pm 1$ we have $d t / d s=0$ and

$$
\frac{d^{2} t}{d s^{2}}=-h \sin \sigma+\frac{f^{\prime}(t)}{f(t)}=\frac{u}{f(t)}
$$

Hence $u<0$ at the maxima of $\left.t\right|_{C}$ and $u>0$ at the minima of $\left.t\right|_{C}$.

A direct computation using equations $(*)$ shows that

$$
J(u)=-K^{\prime}(t) f(t) \cos ^{2} \sigma
$$


and so $J(u)<0$ in the north hemisphere and $J(u)>0$ in the south hemisphere. This implies that the first eigenvalue for the Dirichlet problem of the Jacobi operator is negative in both $C \cap\left\{t \geqslant t_{0} / 2\right\}$ and $C \cap\left\{t \leqslant t_{0} / 2\right\}$. We conclude that $C$ is unstable.

An analysis of the candidates using Theorem 3.3 gives the classification of isoperimetric domains in $M$.

Theorem 3.5. Let $M$ be a rotationally symmetric sphere with an equatorial symmetry, and whose Gauss curvature is a decreasing function of the distance from the poles. Then the isoperimetric domains in $M$ are

(i) geodesic discs centered at the poles, and possibly geodesic discs in regions of constant Gauss curvature about the poles, and their complements,

(ii) annuli symmetric with respect to the equator which are contained in the region $K+h^{2} \leqslant 0$, and their complements,

(iii) nonsymmetric annuli containing the least length meridian which have one boundary component in $K+h^{2}<0$ and the other one in $K+h^{2}>$ 0 , and their complements.

If $K>0$ then (ii) and (iii) cannot happen. If $K \geqslant 0$ then (iii) cannot hold.
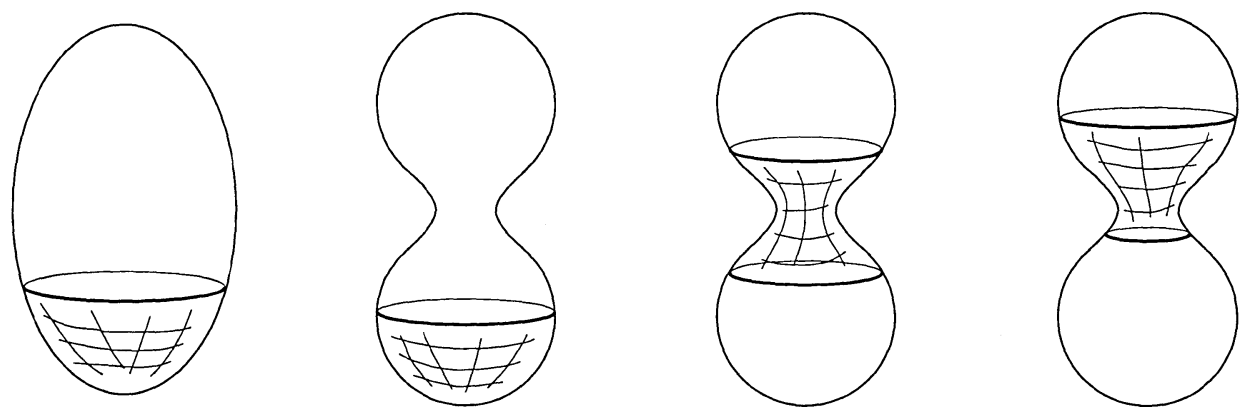

Figure 5: Isoperimetric domains in spheres with curvature increasing from the equator.

Proof. Let $\Omega \subset M$ be an isoperimetric domain. Then $\partial \Omega$ is a curve with constant geodesic curvature $h$ with respect to the inner normal. The com- 
ponents of $\partial \Omega$ are either circles of revolution or the boundaries of constant curvature discs by Theorem 3.3.

Suppose that one component of $\partial \Omega$ is the boundary of a geodesic disc with constant curvature. Replacing $\Omega$ by $M-\Omega$ if necessary we may assume $D \subset \Omega$. Note that in this case $h=h(D)>0$ and that $\lambda_{1}(\partial D)<0$. Let us see that we can replace $\Omega$ by another set bounded by circles of revolution and with no larger perimeter.

If $\partial \Omega=\partial D$ then $\Omega=D$. Lemma 2.7 shows that a geodesic disc $D^{*}$ centered at a pole with the same area as $D$ satisfies $L\left(\partial D^{*}\right) \leqslant L(\partial D)$. Moreover, if $L\left(\partial D^{*}\right)=L(\partial D)$ then both $D$ and $D^{*}$ are contained in a region with constant Gauss curvature around a pole. If $\partial \Omega$ is not connected then any other boundary component $C^{\prime}$ must have $\lambda_{1}\left(C^{\prime}\right)>0$ and so it must be a circle of revolution inside $K+h^{2}<0$. Inside this region the geodesic curvature of circles of revolution is monotonic $\left(h^{\prime}(t)=-\left(K+h^{2}\right)(t)\right)$ and there are exactly two circles with geodesic curvature $\pm h$. The geodesic curvature is positive with respect to the normal pointing to $t=t_{0} / 2$. Then $\Omega$ is the union of $D$ with either a symmetric annulus inside $K+h^{2}<0$ or the union of $D$ with a geodesic disc around a pole. In both cases we can replace $D$ by a geodesic disc $D^{*}$ about a pole with the same area as that of $D$ to obtain a new domain with perimeter less than or equal to $L(\partial \Omega)$. Equality holds if and only if $D$ and $D^{*}$ are contained in a region with constant curvature around a pole. So we may assume that $\Omega$ is bounded by circles of revolution.

So $\partial \Omega=\bigcup_{i=1}^{n} \mathbb{S}^{1} \times\left\{t_{i}\right\}$ with $\left\{t_{i}\right\}$ increasing and $h\left(t_{i}\right)=-h\left(t_{i+1}\right)$, for $i=1, \ldots, n-1$. If $h \neq 0$ then Lemma 3.1 shows that there are at most four circles of revolution in $\partial \Omega$. If $h=0$ then we could have a large number of geodesics of revolution contained in a flat region, but if we have more than two then we can join the annuli they bound in the flat region to obtain a domain with least perimeter, a contradiction.

If two components of $\partial \Omega$ are in $K+h^{2}>0$, then each one has negative first eigenvalue for the Jacobi operator and so $\partial \Omega$ is unstable. Hence at most one component of $\partial \Omega$ is contained in $K+h^{2}>0$. This implies that if $K>0$ then there is at most one boundary component. Moreover if there are four circles in $\partial \Omega$ then two of them are inside $K+h^{2}>0$, as the region where $f^{\prime}>0$ (resp. $f^{\prime}<0$ ) around the south (resp. north) pole is a subset of $K+h^{2}>0$. So $\partial \Omega$ can have at most three boundary components.

If $\partial \Omega$ is connected then $\Omega$ is a geodesic disc around a pole or complement.

When $\partial \Omega$ has two components then $\Omega$ is an annulus or complement. If the annulus is symmetric with respect to the equator then it is contained in the region $K+h^{2} \leqslant 0$ by the stability condition (1.8). If the annulus is 
nonsymmetric then one component must lie in $K+h^{2}>0$ (if both components are inside $K+h^{2} \leqslant 0$ then the annulus is symmetric) and the other one in $K+h^{2}<0$.

Finally assume that $\partial \Omega$ has three boundary components. Then $h>0$ and $\Omega$ is the union of a symmetric annulus contained in the region $K+h^{2} \leqslant 0$ and a geodesic disc around a pole, or its complement. There are annuli with the same area as $\Omega$ and less perimeter than $\Omega$ by [15, Theorem 3.11].

Remark 3.6. If we allow $0<f^{\prime}(0)<1$ all the results in this subsection holds. The metric so obtained is singular at the poles.

Remark 3.7. Assume that $f$ is $C^{1}$ and piecewise $C^{2}$. Then the second variation of length formula is valid at least for a variation by curves meeting the parallels where $K(t)$ is discontinuous at a finite number of points. This is enough to discard the constant geodesic curvature curves which are symmetric with respect to the equator.

Remark 3.8. As in [15] one can show that sometimes annuli have less perimeter than discs of the same area by attaching a long narrow symmetric hyperbolic annulus to two curvature 1 spheres in a $C^{1}$ way. The metric of this surface is $C^{1}$ and piecewise $C^{2}$.

Remark 3.9. Let us see that nonsymmetric annuli can be isoperimetric domains. Attach a hyperbolic annulus to two curvature 1 discs in a $C^{1}$ way. This can be done so that the hyperbolic annulus has strictly less perimeter than a curvature 1 disc of the same area. A sligthly larger symmetric annulus has still less perimeter than the disc of the same area. We deform the annulus by parallels keeping the area enclosed constant until we get a geodesic disc about the pole. The perimeter along this deformation initially decreases since the starting annulus is unstable. As the perimeter of the initial annulus is less than the one of the final disc, there is a stable annulus in the deformation by Lemma 1.7, which cannot be symmetric since it has larger area than the largest symmetric stable annulus.

\subsection{Spheres with curvature decreasing from the equator.}

We assume now that $K(t)$ is a decreasing function of the distance from the equator $t=t_{0} / 2$. The sphere $M$ must have points with positive curvature by Gauss-Bonnet Theorem and so $K\left(t_{0} / 2\right)>0$. On the other hand $K$ could be either positive at the poles (and so $M$ is convex) or negative. In any 
case, $f^{\prime} \geqslant 0$ at the south hemisphere and it vanishes only over the equator. As $f^{\prime}(0)=1, f^{\prime}\left(t_{0} / 2\right)=0,\left(f^{\prime}\right)^{\prime}=-f K$, we have $f^{\prime} \geqslant 0$ and $f^{\prime}=0$ only over the equator.
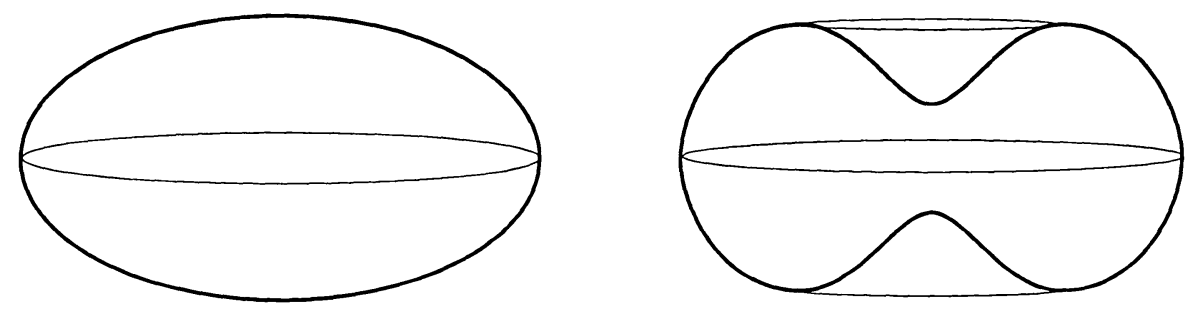

Figure 6: Spheres with curvature decreasing from the equator.

The hypothesis on the Gauss curvature shows that

$$
\left(f^{\prime}\right)^{2}-f f^{\prime \prime} \geqslant 1, \quad \text { on } \quad\left(0, t_{0}\right),
$$

and that equality holds at some region with constant Gauss curvature around the poles.

Lemma 3.2 can be applied to these spheres of revolution to obtain a family of closed embedded nodoids $\left\{C_{T}\right\}, T \in\left(t_{0} / 2, t_{0}\right)$. Under our assumptions we have

Lemma 3.10. Consider the surface $\mathbb{S}^{1} \times\left(0, t_{0}\right)$ with the Riemannian metric $d s^{2}=d t^{2}+f(t)^{2} d \theta^{2}$, with $f\left(t-t_{0}\right)=f(t)$. Assume that $f^{\prime}(t) \geqslant 0$ for $t>t_{0} / 2$ and that $f^{\prime}(t)>0$ for $t<t_{0} / 2$.

Then the family of embedded nodoids $\left\{C_{T}\right\}$, for $T>t_{0} / 2$, foliates an open neighborhood of $p=\left(0, t_{0} / 2\right)$ minus $p$. Moreover the first eigenvalue of the Jacobi operator on $C_{T}$ is negative.

If $M$ is the sphere of revolution described above and $\Omega$ is the hemisphere $\{-\pi / 2 \leqslant \theta \leqslant \pi / 2\}$ then the family of embedded curves $C_{T}$ is defined for $T \in\left(t_{0} / 2, t_{0}\right)$ and it foliates $\Omega-\{p\}$.

Proof. Let us see that the family $\left\{C_{T}\right\}, T>t_{0} / 2$, foliates a punctured neighborhood of $p$. Take $T_{1}>T_{2}>t_{0} / 2$ and let $h_{1}=h\left(T_{1}\right), h_{2}=h\left(T_{2}\right)$. Inequality $h_{1}<h_{2}$ holds by Lemma 3.2. We consider the solutions $\theta_{i}, t_{i}, \sigma_{i}$ to equations $(*)$ with geodesic curvature $h_{i}$ and initial conditions $(\theta, t, \sigma)=$ $\left(0, t_{0} / 2,0\right)$ at $s=0(i=1,2)$. These solutions are translations of the curves $C_{T_{1}}$ and $C_{T_{2}}$ meeting tangentially at $(\theta, t)=\left(0, t_{0} / 2\right)$. 
Near $\left(0, t_{0} / 2\right)$ both curves are graphs over $t$. Writing $\theta_{i}$ and $\sigma_{i}$ as functions of $t$ locally

$$
\left.\frac{d\left(\sin \sigma_{i}\right)}{d t}\right|_{t=t_{0} / 2}=h_{i}
$$

and so $\sin \sigma_{1}(t)<\sin \sigma_{2}(t)$ for $t>t_{0} / 2$ close enough to $t_{0} / 2$ and so $\theta_{1}(t)<$ $\theta_{2}(t)$. Considering now the pieces of both curves where $\sigma_{i} \in(0, \pi / 2)$ and using $\theta$ as parameter we have $t_{1}(\theta)>t_{2}(\theta)$ for $\theta>0$ small enough.

We claim that $t_{1}(\theta), t_{2}(\theta)$ do not meet whenever $\sigma_{i} \in(0, \pi / 2)$. Otherwise there is a first $\theta_{0}$ where they coincide. Since $t_{1}>t_{2}$ in the interval $\left(0, \theta_{0}\right)$ we have $\left(d t_{1} / d \theta\right)\left(\theta_{0}\right) \leqslant\left(d t_{2} / d \theta\right)\left(\theta_{0}\right)$. From $(*)$ we have $\cot \sigma_{1}\left(\theta_{0}\right) \leqslant \cot \sigma_{2}\left(\theta_{0}\right)$ and so $\sigma_{1} \geqslant \sigma_{2}$. On the other hand, as $K(t)$ is decreasing in $\left(t_{0} / 2, t_{0}\right)$, Lemma 1.5 can be applied to $\cos \sigma_{i}$, which are solutions to (1.3), to prove $\cos \sigma_{1}\left(\theta_{0}\right)>\cos \sigma_{2}\left(\theta_{0}\right)$. So $\sigma_{1}\left(\theta_{0}\right)<\sigma_{2}\left(\theta_{0}\right)$. This contradiction shows that $t_{1}(\theta)$ and $t_{2}(\theta)$ never meet when $\sigma_{i} \in(0, \pi / 2)$. If the maximum of $t$ over $C_{T_{i}}$ is achieved at $\theta_{i}, i=1,2$, then another application of Lemma 1.5 shows that $\theta_{2}<\theta_{1}$. From this it follows that $C_{T_{1}}$ and $C_{T_{2}}$ are disjoint. This and the smooth dependence of the solutions to $(*)$ on the initial conditions and on $h$ show that the family $\left\{C_{T}\right\}, T>t_{0} / 2$, gives us a foliation of a punctured neighborhood of $p$.

Let us see now that the first eigenvalue for the Jacobi operator is negative. If we think on $C_{T}$ as a family of curves contracting to $p$, the normal component $\varphi$ of the variational field so obtained is positive. The derivative of the geodesic curvature is positive with respect to this deformation and so $J(\varphi)>0([4])$. Then $I(\varphi)=-\int_{C_{T}} \varphi J(\varphi)<0$ and, by the variational characterization of eigenvalues, $\lambda_{1}\left(C_{T}\right)<0$.

Finally, assume that $\Omega=\{-\pi / 2 \leqslant \theta \leqslant \pi / 2\}$. Then $h(T) \rightarrow 0$ when $T \rightarrow t_{0} / 2$ as $f(T) \rightarrow 0$ when $T \rightarrow t_{0} / 2$. So the curves $C_{T}$ converge to $\partial \Omega$ when $T \rightarrow t_{0}$. This implies that the family $\left\{C_{T}\right\}$ foliates $\Omega-\{p\}$.

Definition 3.11. The foliation constructed in Lemma 3.2 will be called $\mathcal{F}_{M}$. It obviously depends on $\Omega$, but two foliations constructed from different hemispheres are certainly congruent.

For a sphere $M$ in these conditions we have

Theorem 3.12. Let $M$ be a rotationally symmetric sphere with an equatorial symmetry and curvature decreasing as a function of the distance from the equator. Then the only stable embedded curves in $M$ are congruent to the ones in the foliation $\mathcal{F}_{M}$ or to the boundaries of constant curvature discs. 
Proof. Let $C$ be a connected embedded curve with constant geodesic curvature. Assume $C$ is not the boundary of a constant curvature disc. Then it cannot be either a curve touching one pole by Lemma 2.2 nor a stable unduloid by Lemma 2.13. If $C$ is a nodoid then it is symmetric by Lemma 3.4 and so it is congruent to a curve in $\mathcal{F}_{M}$. Meridians are in $\mathcal{F}_{M}$ and circles of revolution are not stable by Lemma 3.3 .

We conclude that a connected embedded curve with constant geodesic curvature is either the boundary of a constant curvature disc or a curve congruent to one in the foliation $\mathcal{F}_{M}$. As both types of curves have $\lambda_{1}<0$ we conclude that an embedded closed stable curve must be connected and the Theorem follows.

Theorem 3.13. Let $M$ be a rotationally symmetric sphere with an equatorial symmetry, and whose Gauss curvature is a decreasing function of the distance from the equator. Then isoperimetric domains in $M$ are the discs enclosed by the curves in the foliation $\mathcal{F}_{M}$ or geodesic discs inside a region with constant curvature around the equator, and their complements.

Proof. Let $\Omega$ be an isoperimetric domain enclosing area $A$. If $\partial \Omega$ is the boundary of a constant curvature disc $D$ we may assume, replacing $\Omega$ by $M-\Omega$ if necessary, that $\Omega=D$.

If $D$ is contained inside a region with constant curvature around the equator, then $D$ is congruent to a disc in the foliation $\mathcal{F}_{M}$. Suppose then that $D$ lies inside a region with constant curvature which does not touch the equator. We have $A(D)<A(M) / 2$. Let $D^{*}$ be a disc bounded by a curve of the foliation $\mathcal{F}_{M}$ with area $A(D)$. Assume that $D$ is contained in the north hemisphere. We claim that $\left.\max t\right|_{D^{*}} \leqslant\left.\max t\right|_{D}$. To show this, let $T=\left.\max t\right|_{D}$ and consider the curves $C_{h}$ with geodesic curvature $h>0$ tangent to $\partial D$ with $\left.\max t\right|_{C_{h}}=T$. For $h>h(\partial D)$ the curves $C_{h}$ are inside $D$. By Lemma 3.2 there is $C_{h}$, with $h<h(D)$ and enclosing a disc $D_{h}$, which gives a closed curve symmetric with respect to the equator. By Lemma 3.14 we have $A\left(D_{h}\right)>A(D)$. So $D^{*} \subset D_{h}$ and the claim follows. The hypotheses of Lemma 2.7 are satisfied and so $L\left(\partial D^{*}\right)<L(\partial D)$.

Lemma 3.14. Consider a rotationally symmetric metric $d s^{2}=d t^{2}+$ $f(t)^{2} d \theta^{2}$. Let $\theta_{i}, t_{i}, \sigma_{i}$, be a solution to $(*)$ with constant geodesic curvature $h_{i}$, and initial conditions $(\theta, t, \sigma)=(0, T, \pi / 2)$, such that $T$ is the maximum value of the $t$-coordinate, $i=1,2$. If the Gauss curvature $K(t)$ is decreasing and $h_{2}>h_{1}>0$ then $t_{2}(\theta)<t_{1}(\theta)$ whenever $d t_{2} / d \theta \neq 0$. 
Proof. Since $h_{2}>h_{1}$ from $(*)$ we have $\left(d \sigma_{2} / d \theta\right)(0)>\left(d \sigma_{1} / d \theta\right)(0)$ and so $t_{2}(\theta)<t_{1}(\theta)$ for $\theta>0$ small. Let us see that inequality $t_{2}(\theta)<t_{1}(\theta)$ is satisfied whenever $d t_{2} / d \theta \neq 0$. Otherwise there is a first $\theta_{0}>0$ such that $t_{1}\left(\theta_{0}\right)=t_{2}\left(\theta_{0}\right)$ and we have $\left(d t_{1} / d \theta\right)\left(\theta_{0}\right) \leqslant\left(d t_{2} / d \theta\right)\left(\theta_{0}\right)$ and so $\cot \sigma_{1}\left(\theta_{0}\right) \leqslant$ $\cot \sigma_{2}\left(\theta_{0}\right)$. As $K(t)$ is decreasing we have $\left(\left(f^{\prime}\right)^{2}-f f^{\prime \prime}\right)\left(t_{2}\right) \geqslant\left(\left(f^{\prime}\right)^{2}-f f^{\prime \prime}\right)\left(t_{1}\right)$ in the interval $\left(0, \theta_{0}\right)$. Lemma 1.5 then shows that $\cos \sigma_{1}\left(\theta_{0}\right)>\cos \sigma_{2}\left(\theta_{0}\right)$. As $\sigma_{i} \in(\pi / 2,3 \pi / 2)$ we have $\sigma_{1}\left(\theta_{0}\right)<\sigma_{2}\left(\theta_{0}\right)$, and so $\cot \sigma_{1}\left(\theta_{0}\right)>\cot \sigma_{2}\left(\theta_{0}\right)$, a contradiction.
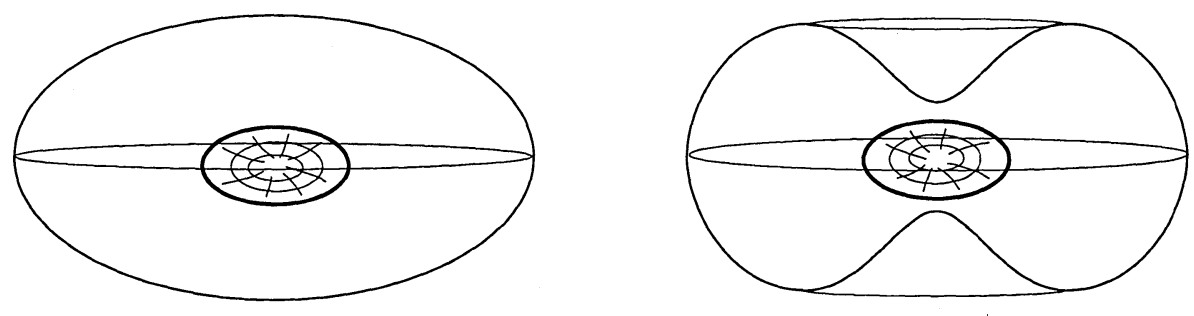

Figure 7: Isoperimetric domains in spheres with curvature decreasing from the equator.

Remark 3.15. If $f^{\prime}(0)>1$ the results in this subsection are still true. Also when $f$ is $C^{1}$ and piecewise $C^{2}$.

Remark 3.16. P. Pansu [20, Lemma 5] has proved that isoperimetric domains with small area in a compact surface are discs close to the maxima of curvature. R. Ye [24] showed that punctured neighborhoods of nondegenerate critical points of the Gauss curvature are foliated by closed embedded curves with constant geodesic curvature.

\section{Projective planes.}

In this section we consider a rotationally symmetric projective plane $M$ whose Gauss curvature is a monotone function of the distance to a given point $p \in M$, the pole of $M$. The orientation covering $S$ of $M$ is a rotationally symmetric sphere with an equatorial symmetry permuting the preimages $p_{1}, p_{2}$ of $p$ (the poles of $S$ ) by the covering map $S \rightarrow M$. The Gauss curvature is a monotone function of the distance $t$ to a pole in $S$ whenever $t \leqslant d\left(p_{1}, p_{2}\right) / 2$. The surface $S$ is of the same type as the ones 
considered in section 3. The surface $M$ minus the pole is the quotient of the sphere $S$ minus its poles by the antipodal map $I(\theta, t)=\left(\theta+\pi, t_{0}-t\right)$.

\subsection{Projective planes with decreasing curvature.}

We now assume that the Gauss curvature is a decreasing function of the distance from the pole $p$. In these conditions we obtain

Theorem 4.1. Let $M$ be a rotationally symmetric projective plane whose Gauss curvature is a decreasing function of the distance from the pole. Then the only connected stable closed embedded curves in $M$ which are two-sided are the circles of revolution and the boundaries of geodesic discs with constant curvature.

Proof. Consider a connected closed embedded curve $C \subset M$ which has constant geodesic curvature and it is two-sided. The lifting of $C$ to $S$ is a connected curve $\widetilde{C} \subset S$. By Theorem 3.3 we have that $\widetilde{C}$ is either

(i) a meridian, or

(ii) a parallel, or

(iii) a closed nodoid symmetric with respect to the equator, or

(iv) a geodesic disc enclosing a region with constant curvature.

If $\widetilde{C}$ is a meridian or $\widetilde{C}$ the equator then $C$ is not two-sided. In the other cases $\widetilde{C}$ is isometric to $C$. So if $\widetilde{C}$ is a symmetric nodoid then $C$ is unstable. If $\widetilde{C}$ encloses a disc $D$ with constant curvature then $I(D) \cap D$ is empty since otherwise the curve $\widetilde{C}=\partial D$ is not embedded. Hence $C$ bounds a constant curvature disc in $M$.

Theorem 4.2. Let $M$ be a rotationally symmetric projective plane with curvature decreasing as a function of the distance from a pole. Then the isoperimetric domains in $M$ are geodesic discs centered at the poles or the boundaries of constant curvature discs inside a region of constant curvature about the pole, and their complements.

Proof. If $\Omega$ is an isoperimetric domain then $\partial \Omega$ consists on stable closed embedded curves which are two-sided. By Theorem 4.1 each component of 
$\partial \Omega$ is either a circle of revolution or the boundary of a constant curvature disc.

Assume that one component $C$ of $\partial \Omega$ is the boundary of a constant curvature disc $D$. We may assume that $D \subset \Omega$. If $\partial \Omega$ is connected then $D=\Omega$ and $D$ is contained in a region with constant curvature around the pole by Lemma 2.7. If there is another connected component $C^{\prime}$ in $\partial \Omega$ then $\lambda_{1}\left(C^{\prime}\right) \geqslant 0$ and so $C^{\prime}$ is a circle of revolution inside $K+h^{2} \leqslant 0$ with positive curvature with respect to the inner normal. Hence $C^{\prime}$ encloses a Möbius band and the pole lies in the complement and we may assume that $\partial \Omega$ is bounded by circles of revolution, replacing $C$ by one of such curves.

If $\partial \Omega$ is the union of circles of revolution of radii $0<t_{1}<\ldots<t_{n}$ then $h\left(t_{i}\right)=-h\left(t_{i+1}\right)$ for $i=1, \ldots, n-1$. As $h$ changes its sign only once we have only two boundary curves in $\partial \Omega$ or an arbitrary number of geodesic in a flat region. In the former case, an annulus bounded by just two geodesic is an optimal solution to the isoperimetric problem. If we have two boundary components then $\Omega$ is the union of a geodesic disc about the pole and a Möbius band inside $K+h^{2} \leqslant 0$. This candidate can be discarded by [15, Lemma 3.2.C].

\subsection{Projective planes with increasing curvature.}

Let us now assume that the Gauss curvature is a increasing function of the distance from the pole $p$. We know that there exists a unique foliation $\mathcal{F}_{S}$ in the orientation covering sphere $S$ of $M$. This foliation induces another one in $M$ which will be denoted by $\mathcal{F}_{M}$. The elements of $\mathcal{F}_{M}$ except the meridian are two-sided circles in $M$ congruent to the ones in $\mathcal{F}_{S}$. They are stable since the circles of $\mathcal{F}_{S}$ are stable (they are solution to the isoperimetric problem in $S$ ).

Theorem 4.3. Let $M$ be a rotationally symmetric projective plane with curvature increasing as a function of the distance from a pole. The only stable closed embedded curves which are two-sided in $M$ are circles congruent to the ones in the foliation $\mathcal{F}_{M}$ or the boundaries of geodesic discs with constant curvature.

Proof. Let $C \subset M$ be a connected stable closed embedded curve which is two-sided. Consider the connected lifting $\widetilde{C}$ of $C$ to $S$. Then $\widetilde{C}$ is either a curve congruent to one in $\mathcal{F}_{S}$ or the boundary of a geodesic disc in $S$. Connectedness of a stable curve follows since the first eigenvalue of each 
connected component is negative.

Theorem 4.4. Let $M$ be a rotationally symmetric projective plane with curvature increasing as a function of the distance from a pole. Then the isoperimetric domains in $M$ are the discs bounded by a curve of the foliation $\mathcal{F}_{M}$ or the boundaries of constant curvature discs inside the region of maximum curvature, and their complements.

Proof. If $\Omega$ is an isoperimetric domain then $\partial \Omega$ is a stable curve with constant geodesic curvature. By Theorem 4.3 we have that $\partial \Omega$ is either a curve congruent to one in $\mathcal{F}_{M}$ or the boundary of a geodesic disc in $M$. If $\Omega$ is a geodesic disc with constant Gauss curvature and it is not contained in the region of maximum curvature then we lift it to $S$ and Theorem 3.13 shows that a disc bounded by some curve in $\mathcal{F}_{S}$ is better. Projecting to $M$ we deduce that a disc bounded by some curve in $\mathcal{F}_{M}$ has less perimeter than $\Omega$.

\section{Cylinders.}

In this section we consider a cylinder $M$ of revolution whose Gauss curvature is a decreasing function of the distance from one end, which we shall call the bottom end, with finite area. The surface $M$ is diffeomorphic to $\mathbb{S}^{1} \times \mathbb{R}$, and the metric $d s^{2}$ equals $d \theta^{2}+f(t)^{2} d t^{2}$. The Gauss curvature $K(t)$ is decreasing. The finite area condition gives

$$
\int_{-\infty}^{t} f(\xi) d \xi<+\infty \text { for all } t
$$

and so

$$
f(t) \sin \sigma-h \int_{-\infty}^{t} f(\xi) d \xi
$$

is a first integral for the system $(*)$.

Lemma 5.1. For the above surface $M$ we have

(i) $K(t)<0$ for all $t$.

(ii) $\lim _{t \rightarrow-\infty} f(t)=\lim _{t \rightarrow-\infty} f^{\prime}(t)=\lim _{t \rightarrow-\infty} f^{\prime \prime}(t)=0$, and so $h(t)>0$ for all $t$. 


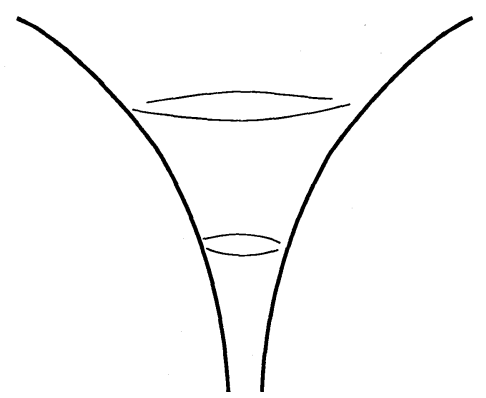

Figure 8: A cylinder with decreasing curvature and a finite area end.

(iii) $\left(f^{\prime}\right)^{2}-f f^{\prime \prime} \leqslant 0$.

Proof. ([15, Corollary 3.5]). Since there are no complete ends with positive curvature and the function $K(t)$ is decreasing we have $K(t) \leqslant 0$. If the Gauss curvature vanishes at some point $t_{0}$ then $K(t)$ vanishes over $\left(-\infty, t_{0}\right]$ and the integral of $f(t)$ over this interval would become infinite. So (i) follows.

Let us see (ii). The finite area condition implies easily that $\lim _{t \rightarrow-\infty} f(t)=$ 0 . Note also that $\lim _{t \rightarrow-\infty} f^{\prime}(t)=0$ : since $f^{\prime}(t)$ is strictly increasing $\left(f^{\prime \prime}>\right.$ $0)$ there exists $\lim _{t \rightarrow-\infty} f^{\prime}(t)$. If this limit is positive then there is $M>0$ such that $f^{\prime}>M$ near $-\infty$ and so $f(t)-f(s)=\int_{s}^{t} f^{\prime}(\xi) d \xi>M(t-s)$. Letting $s \rightarrow-\infty$ we get a contradiction. If the limit is negative (or $-\infty$ ) then $f(t)$ would take negative values near $-\infty$. This proves that $\lim _{t \rightarrow-\infty} f^{\prime}(t)=$ 0 . To see that $\lim _{t \rightarrow-\infty} f^{\prime \prime}(t)=0$ we simply take into account that $K(t)$ converges to a finite limit $K_{-\infty}$ when $t \rightarrow-\infty$ by the monotonicity condition on the curvature, and so $f^{\prime \prime} \rightarrow-K_{-\infty} f=0$ when $t \rightarrow-\infty$. Inequality $h(t)>0$ follows since $f^{\prime \prime}(t)<0$ and $f^{\prime}(t)$ goes to 0 when $t$ goes to $-\infty$. This proves (ii).

Finally (iii) follows since $\left(f^{\prime}\right)^{2}-f f^{\prime \prime}$ goes to 0 when $t \rightarrow-\infty$, and it is a decreasing function.

For these cylinders we have

Theorem 5.2. Consider a rotationally symmetric cylinder $M$ with decreasing curvature and suppose that the end of maximum curvature has finite area. Then the only connected closed embedded curves with constant geodesic curvature are the circles of revolution and the boundaries of geodesic discs with 
constant curvature.

Proof. Let $C$ be a connected closed embedded curve with constant geodesic curvature $h$ in $M$. Then $C$ is either an unduloid or a nodoid. Lemma 2.2 and inequality $\left(f^{\prime}\right)^{2}-f f^{\prime \prime} \leqslant 0$ show that $C$ cannot be an unduloid. If $C$ is a nodoid then $C$ lies inside a region with constant curvature by Lemma 2.2.

Remark 5.3. In fact condition $\left(f^{\prime}\right)^{2}-f f^{\prime \prime} \leqslant 0$, equation 1.3 and Lemma 1.5 imply the non existence of periodic graphs over $\theta$.

Theorem 5.4. Let $M$ be a rotationally symmetric cylinder with decreasing curvature and suppose that the end where the curvature is larger has finite area. Then isoperimetric domains exist on $M$ and they are the finite area regions enclosed by circles of revolution.

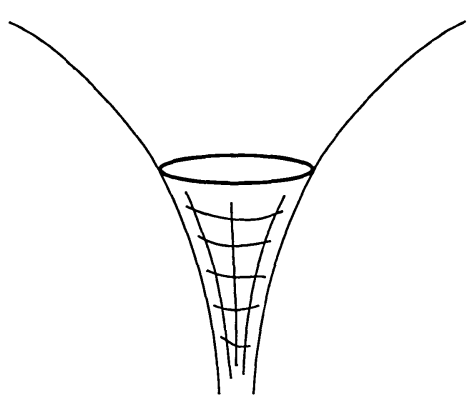

Figure 9: Isoperimetric domains in a cylinder with decreasing curvature and an end of finite area.

Proof. Consider a minimizing sequence $\Omega_{n}$ for area $A$. Then $\Omega_{n}=\Omega_{n}^{c} \cup \Omega_{n}^{d}$ as in Lemma 1.8. Let $\Omega$ be the limit of $\Omega_{n}^{c}$. The divergent sequence $\Omega_{n}^{d}$ approximate the end with smaller curvature since the other one has finite area. Since $K_{\infty}<0$ for this end we repeat the reasoning of Case 2.10 in Theorem 2.8 to conclude that $A(\Omega)=A$. Hence no area loss can happen.

An isoperimetric domain $\Omega$ is bounded by circles of revolution and the boundaries of geodesic discs with constant curvature, with at most one of the former curves since their first eigenvalue is negative. Lemma 5.1 shows that $h(t)>0$ for all $t$. Then $\partial \Omega$ has at most two components, one circle of 
revolution and the boundary of one geodesic disc with constant curvature. If $\partial \Omega$ has two components then $\Omega=B \cup D$, where $B=\left\{t \leqslant t_{1}\right\}$ and $D$ is a geodesic disc with constant curvature $K_{0}$. Let $L_{B}=L(\partial B), L_{D}=L(\partial D)$, and $h>0$ the geodesic curvature of $\partial \Omega$. Consider the function $u$ equal to $-L_{B}$ over $\partial D$ and equal to $L_{D}$ over $\partial B$ has mean zero and the index form applied to $u$ yields

$$
I(u) \leqslant-\left(K_{0}+h^{2}\right)\left(L_{D}^{2} L_{B}+L_{B}^{2} L_{D}\right)<0,
$$

and so $\partial D \cup \partial B$ is unstable. We conclude that an isoperimetric domain is either an annulus $\left\{t \leqslant t_{1}\right\}$ or a geodesic disc with constant curvature. Let us see that, for the same enclosed area, the annulus has least perimeter.

Consider an annulus $B=\left\{t \leqslant t_{1}\right\}$ and a geodesic disc $D$ with constant curvature $K_{0}$, both enclosing area the same area. Then $D$ cannot be contained in $B$ and so the total curvature of $B$ is greater than or equal to the one of $D$. Writing the derivative of the perimeters of both sets as functions of the area $A$ we have

$$
\begin{aligned}
& \frac{d L^{2}(\partial D)}{d A}=2 h(\partial D) L(\partial D)=2\left(2 \pi-\int_{D} K d M\right), \\
& \frac{d L^{2}(\partial B)}{d A}=2 h(\partial B) L(\partial B)=-2 \int_{B} K d M .
\end{aligned}
$$

As the total curvature of $B$ is greater than or equal to the one of $D$ we have $d L^{2}(\partial B) / d A<d L^{2}(\partial D) / d A$ and so $L(\partial B)<L(\partial D)$.

Example 5.5. We now show by example that in a complete rotationally symmetric annulus with increasing curvature some circles of revolution can be unstable. The arguments used to prove Theorem 5.2 do not cover this case and indeed we believe that closed embedded unduloids exist in this surface. We fix $t_{0}>0$ and consider the function $f: \mathbb{R} \rightarrow \mathbb{R}$ given by

$$
f(t)= \begin{cases}\exp (a t), & t \leqslant t_{0}, \\ c t, & t \geqslant t_{0},\end{cases}
$$

which is $C^{1}$ if $a=t_{0}^{-1}, c=\exp (1) t_{0}^{-1}$. Over the annulus $\mathbb{S}^{1} \times \mathbb{R}$ the Gauss curvature of the metric $d t^{2}+f(t)^{2} d \theta^{2}$ is increasing in $t$ and equals

$$
K(t)= \begin{cases}-a^{2}, & t<t_{0} \\ 0, & t>t_{0}\end{cases}
$$


The function

$$
\left(\left(f^{\prime}\right)^{2}-f f^{\prime \prime}\right)(t)= \begin{cases}0, & t<t_{0}, \\ c^{2}, & t>t_{0},\end{cases}
$$

takes values larger than 1 when $c>1$, and so the circles of revolution $\mathbb{S}^{1} \times\left\{t_{1}\right\}$ are unstable for $t_{1}>t_{0}$. It is an open question how the isoperimetric domains look like in this surface.

The bottom end $(t \rightarrow-\infty)$ of the above metric has finite area, and the top end (the one with the largest curvature) has infinite area. So this surface also shows that the hypothesis of finite area is necessary to obtain the bound $\left(f^{\prime}\right)^{2}-f f^{\prime \prime} \leqslant 0$ in Lemma 5.1.

Additional examples of complete rotationally symmetric cylinders of monotone curvature with both ends of infinite area and with unstable circles of revolution are provided by the functions,

$$
f(t)= \begin{cases}\lambda \cosh (a t), & t \leqslant t_{0}, \\ \lambda c \sinh (b t), & t \geqslant t_{0},\end{cases}
$$

where $t_{0}>0$ is fixed, $a, b, c$ are choosen so that $f(t)$ is $C^{1}$, and $\lambda>0$ is constant large enough so that $\left(f^{\prime}\right)^{2}-f f^{\prime \prime}>1$.

\section{References.}

[1] J.L. Barbosa, M. do Carmo and J. Eschenburg, Stability of hypersurfaces with constant mean curvature in Riemannian manifolds, Math. Z., 197 (1988), 123-138.

[2] C. Bavard and P. Pansu, Sur le volume minimal de $\mathbb{R}^{2}$, Ann. Sci. École Norm. Sup., 19(4) (1986), 479-490.

[3] I. Benjamini and J. Cao, A new isoperimetric comparison theorem for surfaces of variable curvature, Duke Math. J., 85(2) (1996), 359-397.

[4] I. Chavel, Eigenvalues in Riemannian Geometry, Pure and Applied Mathematics, 115, Academic Press, Inc., Orlando, Fla., 1984.

[5] I. Chavel, Riemannian Geometry-a modern introduction, Cambridge Tracts in Mathematics, 108, Cambridge University Press, Cambridge, 1993. 
[6] C. Delaunay, Sur la surface de revolution dont la courbure moyenne est constante, J. Math. Pures Appl., 16 (1841), 309-321.

[7] M. do Carmo, Differential geometry of curves and surfaces, PrenticeHall, Inc., 1976.

[8] F. Fiala, Le problème des isopérimètres sur les surfaces ouvertes à courbure positive, Comment. Math. Helv., 13 (1941), 293-346.

[9] M. Giaquinta and S. Hildebrandt, Calculus of Variations I: The Lagrangian formalism, Grundlehren Math. Wiss., 310, Springer Verlag, Berlin, 1996.

[10] E. Giusti, Minimal Surfaces and Functions of Bounded Variation, Monographs in Mathematics, 80, Birkhäuser, Verlag, Basel-Boston, 1984.

[11] M. Grayson, Shortening embedded curves, Ann. of Math., 129(2) (1989), 71-111.

[12] P. Hartman, Geodesic parallel coordinates in the large, Amer. J. Math., 8 (1964), 705-727.

[13] P. Hartman, Ordinary differential equations, John Wiley \& Sons, Inc., New York-London-Sydney, 1964.

[14] H. Howards, M. Hutchings and F. Morgan, The isoperimetric problem on surfaces, Amer. Math. Monthly, 106(5) (1999), 430-439.

[15] H. Howards, M. Hutchings and F. Morgan, The isoperimetric problem on surfaces of revolution of decreasing Gauss curvature, Trans. Amer. Math. Soc., 352(11) (2000), 4889-4909.

[16] J. Kazdan and F. Warner, Surfaces of revolution with monotonic increasing curvature and an application to the equation $\Delta u=1-K e^{2 u}$ on $S^{2}$, Proc. Amer. Math. Soc., 32 (1972), 139-141.

[17] S. Montiel, Unicity of constant mean curvature hypersurfaces in foliated Riemannian manifolds, Indiana Univ. Math. J., 48(2) (1999), 711-748.

[18] R. Osserman, The isoperimetric inequality, Bull. Amer. Math. Soc. (N.S.), 84(6) (1978), 1182-1238.

[19] R. Osserman, Bonnesen-style isoperimetric inequalities, Amer. Math. Monthly, 86(1) (1979), 1-29. 
[20] P. Pansu, Sur la régularité du profil isopérimétrique des surfaces riemannienes compactes, Ann. Inst. Fourier (Grenoble), 48(1) (1998), 247-264.

[21] M. Ritoré, Constant geodesic curvature curves and isoperimetric domains in rotationally symmetric surfaces: tori, Klein bottles and symmetric cylinders, in preparation.

[22] E. Schmidt, Über eine neue Methode zur Behandlung einer Klasse isoperimetrischer Aufgaben im Grossen, Math. Z., 47 (1942), 489-642.

[23] P. Topping, The isoperimetric inequality on a surface, Manuscripta Math., 100(1) (1999), 23-33.

[24] R. Ye, Foliation by constant mean curvature spheres, Pacific J. Math., 147(2) (1991), 381-396.

Departamento de Geometría y Topología

Universidad DE Granada

E-18071 GRANADA

ESPAÑA

E-mail address: ritore@ugr.es

RECEIVED July 7, 1999. 